\title{
Evaluating Force Field Performance in Thermodynamic Calculations of Cyclodextrin Host-Guest Binding: Water Models, Partial Charges, and Host Force Field Parameters
}

\author{
Niel M. Henriksen ${ }^{\circledR}$ and Michael K. Gilson*이 \\ Skaggs School of Pharmacy and Pharmaceutical Sciences, University of California San Diego, La Jolla, California 92093-0736, United \\ States
}

\section{Supporting Information}

\begin{abstract}
Computational prediction of noncovalent binding free energies with methods based on molecular mechanical force fields has become increasingly routine in drug discovery projects, where they promise to speed the discovery of small molecule ligands to bind targeted proteins with high affinity. Because the reliability of free energy methods still has significant room for improvement, new force fields, or modifications of existing ones, are regularly introduced with the aim of improving the accuracy of molecular simulations. However, comparatively little work has been done to systematically assess how well force fields perform, particularly in relation to the calculation of binding affinities. Hardware advances have made these calculations feasible, but comprehensive force field assessments for protein-ligand sized systems still remain costly. Here, we turn to cyclodextrin host-guest systems, which feature many hallmarks of protein-ligand binding interactions but are generally much

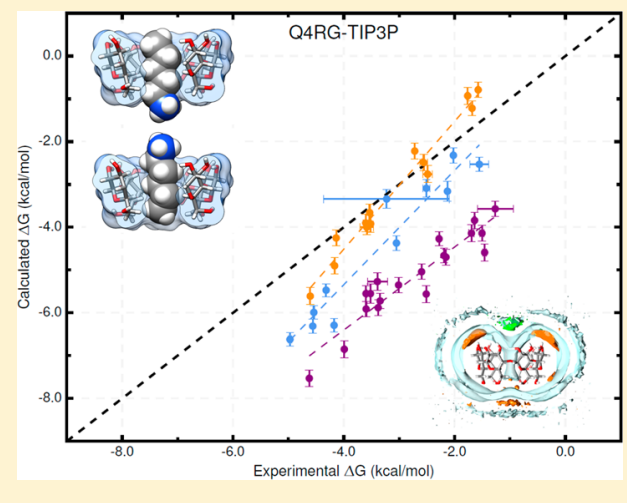
more tractable due to their small size. We present absolute binding free energy and enthalpy calculations, using the attach-pull-release (APR) approach, on a set of 43 cyclodextrin-guest pairs for which experimental ITC data are available. The test set comprises both $\alpha$ - and $\beta$-cyclodextrin hosts binding a series of small organic guests, each with one of three functional groups: ammonium, alcohol, or carboxylate. Four water models are considered (TIP3P, TIP4Pew, SPC/E, and OPC), along with two partial charge assignment procedures (RESP and AM1-BCC) and two cyclodextrin host force fields. The results suggest a complex set of considerations when choosing a force field for biomolecular simulations. For example, some force field combinations clearly outperform others at the binding enthalpy calculations but not for the binding free energy. Additionally, a force field combination which we expected to be the worst performer gave the most accurate binding free energies - but the least accurate binding enthalpies. The results have implications for the development of improved force fields, and we propose this test set, and potential future elaborations of it, as a powerful validation suite to evaluate new force fields and help guide future force field development.
\end{abstract}

\section{INTRODUCTION}

Accurate binding affinity prediction using molecular mechanics force fields has long been a goal of the computer-aided drug design (CADD) community. Molecular mechanics offers lower computational cost than quantum mechanical approaches, which makes it tractable on systems relevant to drug design (i.e., full proteins), while still maintaining an explicit atom description of the system, which is likely necessary to produce accurate results. However, choosing which force field to use can be a daunting task, as new options are constantly being introduced. There are traditional variants with long development trees, notably OPLS, ${ }^{1,2}$ AMBER, ${ }^{3}$ CHARMM, ${ }^{4,5}$ and GROMOS $^{6}$ as well as newer or more exotic force fields, such as Kirkwood-Buff FF, ${ }^{7}$ CHARMM Drude, ${ }^{8,9}$ induced dipole, ${ }^{10,11}$ AMOEBA, ${ }^{12} \mathrm{GEM}^{*},{ }^{13}$ Jorgensen's atoms-in-molecules approach, ${ }^{14}$ QMDFF, ${ }^{15}$ ReaxFF, ${ }^{16}$ X-Pol, ${ }^{17}$ and many others. These options pertain primarily to the treatment of the protein and ligand, but there are also several frequently used water models such as TIP3P, ${ }^{18}$ TIP4Pew, $^{19}$ and SPC $/ E,{ }^{20}$ and new ones are being added, such as OPC, ${ }^{21}$ TIP3P-FB, ${ }^{22}$ TIP4PFB, ${ }^{22}$ TIP4P-D, ${ }^{23}$ and iAMOEBA. ${ }^{24}$ However, tests of the ability of these force fields to replicate experimental data are, arguably, sparse.

Perhaps the experimental data type most widely used to evaluate force fields today is the free energy of hydration of small molecules, ${ }^{2,25,26}$ with some use also of nucleic acid structure $^{27,28}$ and protein structure. ${ }^{5,23,29,30}$ Indeed, although the accurate calculation of binding affinities is a core goal of CADD methods, few studies have used noncovalent binding thermodynamic data to test force fields in the context of explicit solvent methods. (Two recent studies using cyclodextrin-guest binding free energies as tests in the context of implicit solvent models deserve mention, however. ${ }^{31,32}$ ) In order to test the accuracy of force fields for the calculation of binding thermodynamics, it would perhaps appear ideal to use

Received: April 4, 2017

Published: July 11, 2017 


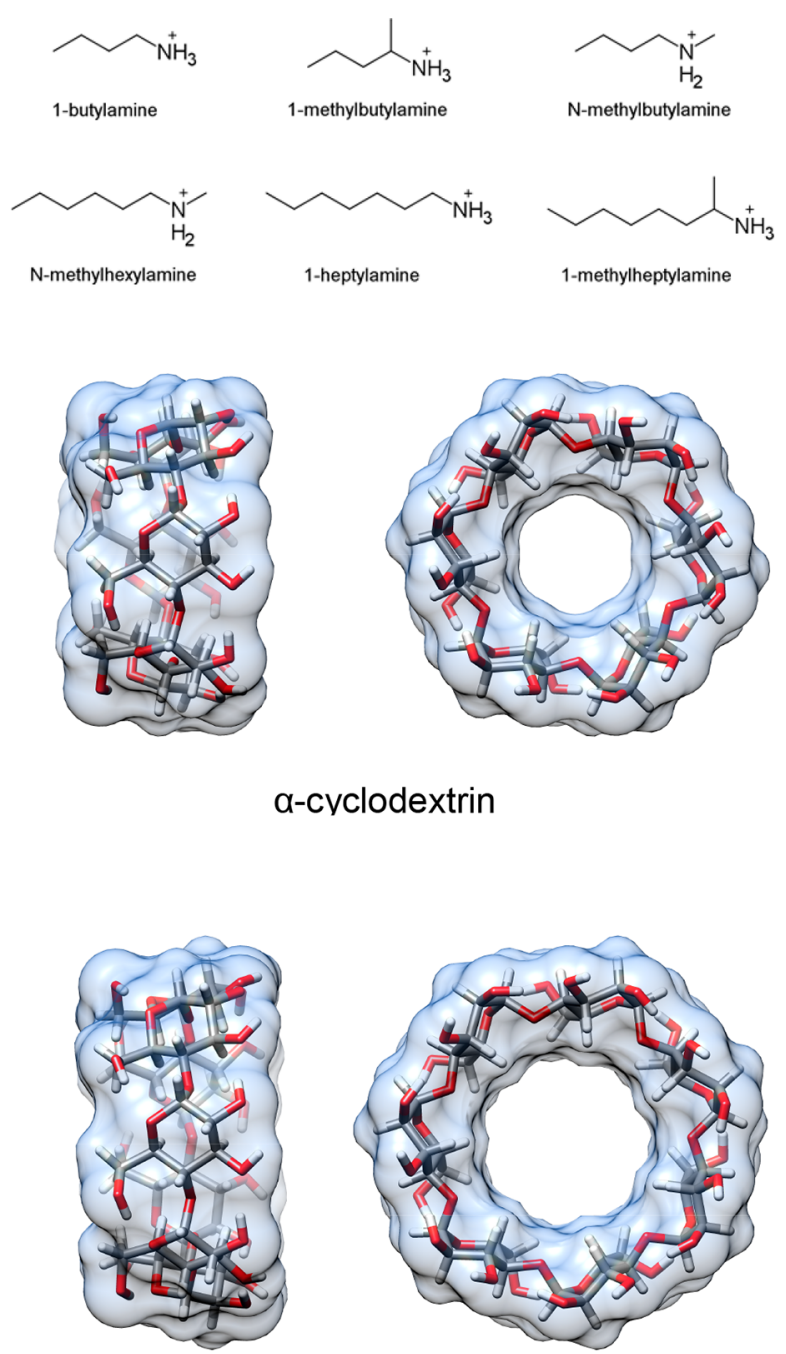

$\beta$-cyclodextrin
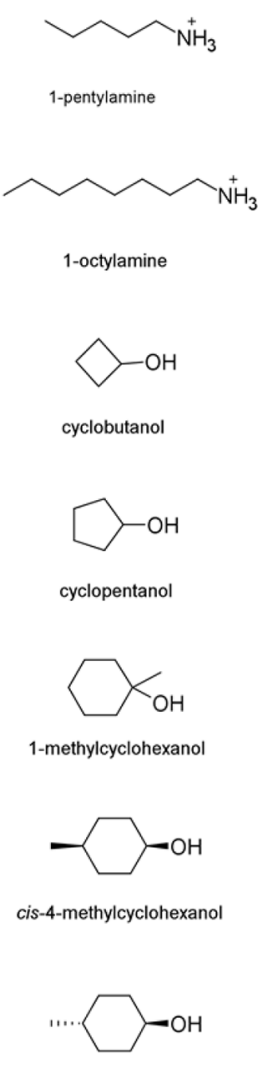

trans-4-methylcyclohexanol

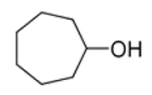

cycloheptanol

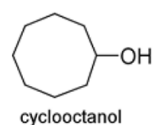

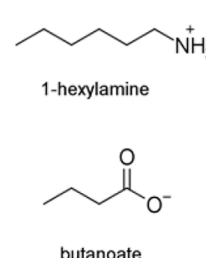<smiles>CCCCC(=O)OC</smiles>

pentanoate<smiles>CCCCCC(=O)[O-]</smiles>

hexanoate<smiles>CCC/C=C/C(=O)[O-]</smiles>

trans-2-hexenoate<smiles></smiles>

trans-3-hexenoate<smiles>CCCCCCC(=O)[O-]</smiles><smiles>C=CCCCCC(=O)OC</smiles>

6-heptenoate<smiles>CCCCCCCC(=O)[O-]</smiles><smiles>CCCCCC(C)N</smiles>

1-methylhexylamine<smiles>O=C([O-])c1ccccc1</smiles>

benzoate

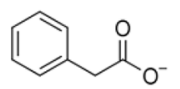

phenylacetate

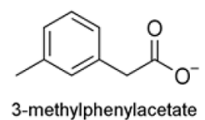

$1+{ }^{\circ}$

4-methylphenylacetate

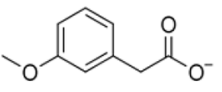

3-methoxyphenylacetate
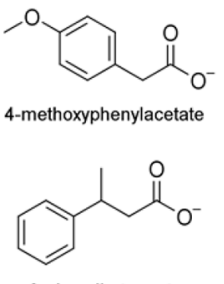

3-phenylbutanoate

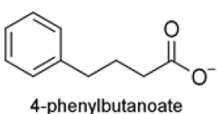

Figure 1. Structures of the hosts $(\alpha$-and $\beta$-cyclodextrin) and guests in this study. The 43 specific host-guest binding pairs and their assigned abbreviations are given in Table 1 . Guest names match the convention in Tables 1 and 2 of Rekharsky et al. ${ }^{37}$

protein-ligand data, since these are most directly relevant to the challenge of drug design. However, the accuracy of such calculations depends not only on the choice of force field but also on setup choices, like the protonation states of binding site residues; and the possibility of slow protein motions means that it is difficult to be confident that the calculations are well converged. As a consequence, the level of agreement with experiment may not reliably reflect the accuracy of the force field. In recent years, host-guest systems have emerged as valuable models for testing the accuracy of simulation methods in the context of noncovalent binding. ${ }^{33-35}$ Host-guest systems feature many characteristics of protein-ligand binding (desolvation, $\mathrm{H}$-bonds, rotational restriction), while being small enough for precise thermodynamic calculations to be tractable. In addition, unlike proteins, one can often have high confidence in the assignment of protonation states at a given $\mathrm{pH}$. These characteristics make it easier to attribute the level of accuracy to the choice of force field. It is worth noting that, although blinded community challenges like SAMPL ${ }^{33-35}$ now often include host-guest systems, interpreting the results can be complicated by the relatively small number of cases and the lack of systematic testing of force field variations.

Here, in order to inform our ongoing effort to incorporate binding calculations into force field development, ${ }^{36}$ we report binding free energy and enthalpy results for 43 host-guest pairs, computed using several different water models, partial charge assignment methods, and host force field parameters. The experimental study from which we draw these systems ${ }^{37}$ makes for an ideal test case: 1) it contains several compounds of various sizes for each of three guest classes, including ammoniums, cyclic alcohols, and carboxylates; 2) these functional groups are attached to both linear and cyclic aliphatic scaffolds, as well as phenyl groups; and 3) these guests are tested with two closely related host molecules of different sizes, $\alpha$-cyclodextrin $(\alpha \mathrm{CD})$ and $\beta$-cyclodextrin $(\beta \mathrm{CD})$. In addition, the experimental data includes high quality ITC measurements of both binding free energy and binding enthalpy, along with NMR data which reports chemical shifts of both the host and guest.

Our results provide insight for researchers wondering how changes in water model, partial charge assignment method, and 
Table 1. Forty-Three Host-Guest Binding Pairs Studied in This Work ${ }^{c}$

\begin{tabular}{|c|c|c|c|c|c|}
\hline HG ID & host & guest [charge] & HG ID & host & guest [charge] \\
\hline a-bam & $\alpha \mathrm{CD}$ & 1-butylamine $[+1]$ & b-chp & $\beta \mathrm{CD}$ & cycloheptanol $[0]$ \\
\hline a-nmb & $\alpha \mathrm{CD}$ & n-methylbutylamine $[+1]$ & $\mathrm{b}-\operatorname{coc}$ & $\beta \mathrm{CD}$ & cyclooctanol $[0]$ \\
\hline$a-m b a$ & $\alpha \mathrm{CD}$ & 1-methylbutylamine $[+1]^{a}$ & a-but & $\alpha \mathrm{CD}$ & butanoate $[-1]$ \\
\hline a-pam & $\alpha \mathrm{CD}$ & 1-pentylamine $[+1]$ & a-pnt & $\alpha \mathrm{CD}$ & pentanoate $[-1]$ \\
\hline a-ham & $\alpha \mathrm{CD}$ & 1-hexylamine $[+1]$ & a-hex & $\alpha \mathrm{CD}$ & hexanoate[-1] \\
\hline a-nmh & $\alpha \mathrm{CD}$ & n-methylhexylamine $[+1]$ & $\mathrm{a}-\mathrm{hx} 2$ & $\alpha \mathrm{CD}$ & trans-2-hexenoate $[-1]$ \\
\hline a-mha & $\alpha \mathrm{CD}$ & 1-methylhexylamine $[+1]^{a}$ & $\mathrm{a}-\mathrm{hx} 3$ & $\alpha \mathrm{CD}$ & trans-3-hexenoate $[-1]$ \\
\hline a-hpa & $\alpha \mathrm{CD}$ & 1-heptylamine $[+1]$ & a-hep & $\alpha \mathrm{CD}$ & heptanoate[-1] \\
\hline a-mhp & $\alpha \mathrm{CD}$ & 1-methylheptylamine $[+1]^{b}$ & a-hp6 & $\alpha \mathrm{CD}$ & 6 -heptenoate $[-1]$ \\
\hline a-oam & $\alpha \mathrm{CD}$ & 1-octylamine $[+1]$ & a-oct & $\alpha \mathrm{CD}$ & octanoate $[-1]$ \\
\hline b-ham & $\beta \mathrm{CD}$ & 1-hexylamine $[+1]$ & b-pnt & $\beta \mathrm{CD}$ & pentanoate $[-1]$ \\
\hline b-mha & $\beta \mathrm{CD}$ & 1-methylhexylamine $[+1]^{a}$ & b-hex & $\beta \mathrm{CD}$ & hexanoate $[-1]$ \\
\hline b-oam & $\beta \mathrm{CD}$ & 1-octylamine $[+1]$ & b-hep & $\beta \mathrm{CD}$ & heptanoate[-1] \\
\hline $\mathrm{a}-\mathrm{cbu}$ & $\alpha \mathrm{CD}$ & cyclobutanol $[0]$ & b-ben & $\beta \mathrm{CD}$ & benzoate $[-1]$ \\
\hline a-cpe & $\alpha \mathrm{CD}$ & cyclopentanol $[0]$ & b-pha & $\beta \mathrm{CD}$ & phenylacetate $[-1]$ \\
\hline a-chp & $\alpha \mathrm{CD}$ & cycloheptanol $[0]$ & b-mp3 & $\beta \mathrm{CD}$ & 3-methylphenylacetate[-1] \\
\hline $\mathrm{a}-\operatorname{coc}$ & $\alpha \mathrm{CD}$ & cyclooctanol $[0]$ & b-mp4 & $\beta \mathrm{CD}$ & 4-methylphenylacetate[-1] \\
\hline b-cbu & $\beta \mathrm{CD}$ & cyclobutanol $[0]$ & b-mo3 & $\beta \mathrm{CD}$ & 3-methoxyphenylacetate[-1] \\
\hline b-cpe & $\beta \mathrm{CD}$ & cyclopentanol $[0]$ & b-mo4 & $\beta \mathrm{CD}$ & 4-methoxyphenylacetate[-1] \\
\hline b-mch & $\beta \mathrm{CD}$ & 1-methylcyclohexanol $[0]$ & b-pb3 & $\beta \mathrm{CD}$ & 3-phenylbutanoate[-1] \\
\hline $\mathrm{b}-\mathrm{m} 4 \mathrm{c}$ & $\beta \mathrm{CD}$ & cis-4-methylcyclohexanol[0] & b-pb4 & $\beta \mathrm{CD}$ & 4-phenylbutanoate[-1] \\
\hline b-m $4 \mathrm{t}$ & $\beta \mathrm{CD}$ & trans-4-methylcyclohexanol $[0$ & & & \\
\hline
\end{tabular}

${ }^{a}$ Only the $R$ enantiomer was considered. ${ }^{b}$ Only the $S$ enantiomer was considered. ${ }^{c}$ The formal charges used in the calculations are listed in brackets. See also Figure 1. Guest names match conventions in Tables 1 and 2 of Rekharsky et al. ${ }^{37}$

other force parameters influence binding results. More specifically, they show that none of the force field combinations we tested proved to be superior to the others for computing both binding free energy and binding enthalpy. They reveal more than one mechanism through which entropy-enthalpy compensation can operate in binding reactions involving CDs. Finally, the test set can serve as a critical component of an overall force field optimization strategy, providing important feedback on how force field changes affect the accuracy of binding calculations. ${ }^{38}$

\section{METHODS}

Host-Guest Systems. We studied the binding thermodynamics for 43 host-guest pairs from the Rekharsky et al. study. ${ }^{37}$ These pairs consisted of either $\alpha \mathrm{CD}$ or $\beta \mathrm{CD}$ as the host and an ammonium, carboxylate, or cyclic alcohol guest. Structures are provided in Figure 1 and are listed in Table 1, along with a brief ID used in other data tables. Note that only one stereoisomer was considered for the 1-methylammonium guests as it was unclear from the experimental details whether a mixture was used and, if so, what ratio was used. The differences in binding between enantiomers of these guests is not expected to exceed the estimated uncertainty in our calculations. $^{39}$

Thermodynamic Calculations. We used the attach-pullrelease (APR) method ${ }^{40}$ to compute the absolute binding free energies. Briefly, this entails a series of umbrella sampling simulations, in which restraints controlling the host-guest complex are incrementally activated, then used to pull the host and guest apart, and finally released in such a way as to leave the guest at standard concentration. The attachment phase consisted of 15 independent windows with nonuniform spacing in the force constant that concentrated windows on the lower force constant domain. This spacing improves the precision of thermodynamic integration calculations. The pulling phase consisted of 45 independent windows, in which a distance restraint controlling the host-guest distance pulled the guest $18 \AA$ away from the host in uniform increments of $0.4 \AA$. A set of dihedral restraints was added to the $\mathrm{CD}$ host during the attachment phase (on atoms $\mathrm{OS}_{n}-\mathrm{Cl}_{n}-\mathrm{O} 1_{n}-\mathrm{C} 4_{n+1}$ and $\mathrm{Cl}_{n}-\mathrm{O} 1_{n}-$ $\left.\mathrm{C}_{n+1}-\mathrm{CS}_{n+1}\right)$ in order to limit the conformational flexibility of the host, thereby improving convergence during the pulling phase. These restraints were accounted for in the overall free energy calculation by including a release phase calculation in which the conformational restraints were gradually turned off over 15 independent windows, with the same force constant spacing as the attachment phase, in the absence of any guest. Finally, in order to compare with experimental values in which standard state of the guest is defined as $1 \mathrm{M}$, we analytically compute the work to move the unbound guest from the restricted volume enforced by the APR restraints to $1 \mathrm{M}$ (i.e., 1 molecule/1660 $\AA^{3}$ ). See Henriksen et al. for a complete description of this calculation. ${ }^{40}$

For simple guest molecules, with one polar functional group, there are generally two possible binding modes in the CD cavity: one with the polar group oriented out of the $C D$ opening with primary alcohols and one with the polar group oriented out of the wider opening with secondary alcohols (Figure 2). However, experimental data report binding free energy and enthalpy values based on a Boltzmann-weighted ensemble of these two orientations. In order to compare with experiment, we separately compute and appropriately combine the binding free energy and enthalpy for each orientation, as previously described. ${ }^{40}$

For some force field combinations and/or guest orientations, the binding affinity is so small or the barrier to entry/exit from the cavity is so low, that the guest could frequently leave the binding cavity during the early stages of the attachment step. This introduces a large statistical noise into the procedure, while the guest wanders around the simulation box in the bulk 

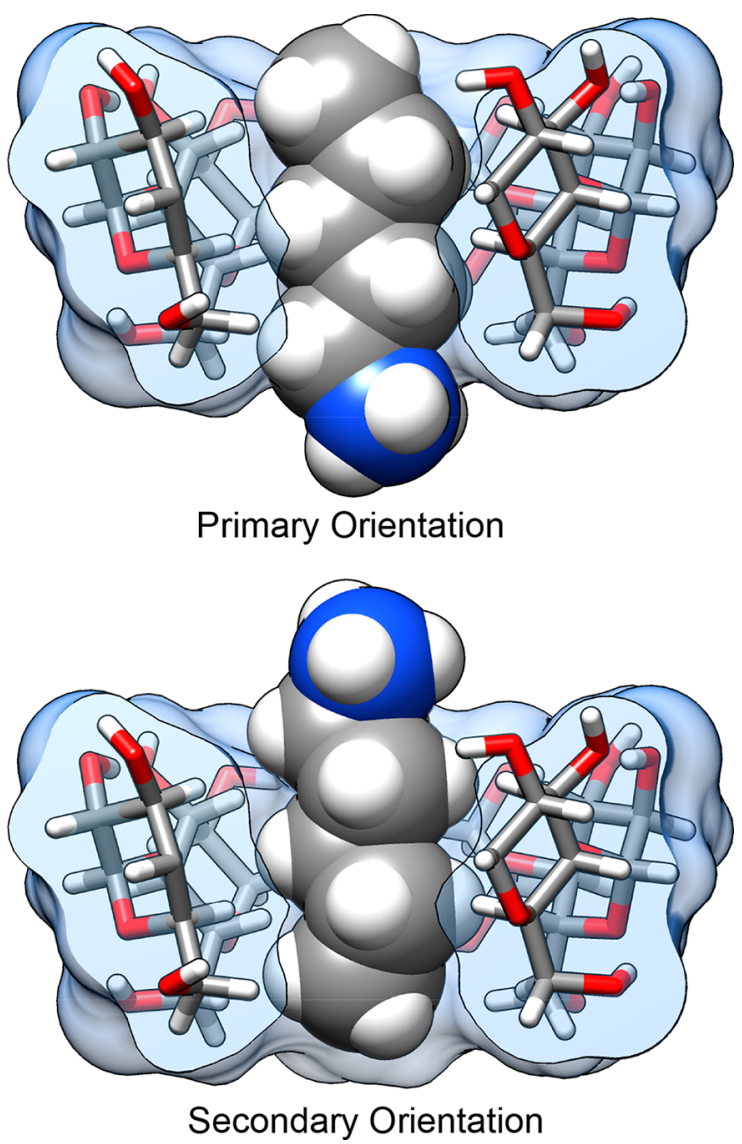

Figure 2. Example of the pentylammonium guest bound to the $\alpha \mathrm{CD}$ host in both the primary (top) and secondary (bottom) orientations. The name of the orientation refers to the positioning of the guest polar group at the $\mathrm{CD}$ opening with either the primary alcohol groups or secondary alcohol groups of the cyclodextrin. One of the glucose monomers in the cyclodextrin has been removed for visualization purposes.

solvent. To prevent this behavior, a hard "wall restraint" is introduced during the attachment phase, which acts as a reflective sphere around the $\mathrm{CD}$ host and prevents the guest from completely entering bulk solvent. The wall restraints were defined as one-sided harmonic restraints between the C6 and $\mathrm{O} 3$ atoms of the $\mathrm{CD}$ host and a single atom of the guest. The distances beyond which the harmonic potential was nonzero were 12.3 and $13.5 \AA$ for $\alpha \mathrm{CD}$ and $\beta \mathrm{CD}$, respectively. The force constant for these restraints was $50 \mathrm{kcal} / \mathrm{mol}-\AA^{2}$.

The binding free energy was computed via a thermodynamic integration approach, using Python scripts developed by our $\mathrm{lab}$, in which the restraint force constants were scaled by the $\lambda$ parameter in the attachment and release phases, and the distance restraint target value was the $\lambda$ parameter in the pulling phase. For comparison, we also computed the free energy with the MBAR method. ${ }^{41}$ The binding enthalpy was computed by subtracting the average potential energy of the last window of the release phase, in which the guest has been pulled far away from the host, from the mean energy of the first window of the attachment phase, in which restraint force constants are off. The uncertainties for average properties calculated in each window (e.g., restraint coordinate values and potential energies) were estimated with the blocking method ${ }^{42}$ and propagated into the final reported values using bootstrapping for the thermodynamic integration calculations or addition in quadrature for the binding enthalpy calculations. The binding entropy can subsequently be deduced by subtraction of the binding enthalpy from the binding free energies, and we report these values in the Supporting Information tables. A similar approach to determining cyclodextrin-guest binding thermodynamics has been reported previously. ${ }^{43}$

A complete description of the APR method, thermodynamic analysis, and uncertainty estimation is provided in our earlier paper. $^{40}$ The approach in this paper matches the $\beta \mathrm{CD}-\mathrm{Hex}_{\mathrm{HMR}}$ simulations referenced in that work.

Force Field Parameters. The simulation system for each host-guest pair consisted of the following: 2000 and 2210 water molecules for $\alpha \mathrm{CD}$ and $\beta \mathrm{CD}$ hosts, respectively; appropriate $\mathrm{Na}^{+}$or $\mathrm{Cl}^{-}$ions to neutralize the total system; additional $50 \mathrm{mM} \mathrm{NaCl}$ or $\mathrm{NaH}_{2} \mathrm{PO}_{4} / \mathrm{Na}_{2} \mathrm{HPO}_{4}$ buffer to match experimental conditions; ${ }^{37}$ and a single $\mathrm{CD}$ host and guest molecule. As part of this investigation, we examined the influence of water model, ion parameters, partial charge assignment methods, and the $\mathrm{CD}$ force field. Four water models were studied: TIP3P, ${ }^{18}$ TIP4Pew, ${ }^{19}$ SPC/E, ${ }^{20}$ and OPC. ${ }^{21}$ Ion parameters for $\mathrm{Na}^{+}$and $\mathrm{Cl}^{-}$were taken from Joung and Cheatham's work, ${ }^{44}$ in which the parameters were tuned for each water model, except for simulations with the OPC water model, for which we tried both Joung and Cheatham's TIP4Pew ion parameters as well as parameters tuned specifically for matching hydration energies in OPC (distributed with AMBER; see Sections 3.5 and 3.6 in the AMBER 16 manual). Results with the $\mathrm{NaH}_{2} \mathrm{PO}_{4} / \mathrm{Na}_{2} \mathrm{HPO}_{4}$ buffer are reported only for the OPC water model, as these ions were observed to aggregate unrealistically into ordered structures when used with other water models. The parameters for these phosphate ions were based on those suggested by Steinbrecher et al. ${ }^{45}$ The CD host molecules were parametrized with either the Q4MD-CD force field, ${ }^{46}$ which uses RESP derived charges and draws many Lennard-Jones and valence parameters from GLYCAM04, ${ }^{47-49}$ or a crude "minimal-effort" version we created as a control. In the latter approach, a single glucose molecule, with methoxy caps on the $\mathrm{O} 1$ and $\mathrm{O} 4$ alcohols, was parametrized with AM1-BCC ${ }^{50,51}$ partial charges and GAFF v1. $7^{52}$ Lennard-Jones and valence parameters, using AMBER's antechamber and parmchk2 utilites. Guest molecules used either RESP partial charges computed with the R.E.D. Server ${ }^{53}$ or AM1-BCC charges computed with antechamber. Guest Lennard-Jones and valence parameters were obtained from GAFF v1.7. A complete list of the force field combinations tested is provided in Table 2 .

Simulation Settings. All simulations were performed with either the AMBER 14 or $16^{3}$ molecular dynamics software. For each simulation window, the entire system was rebuilt using AMBER's tleap utility, thus ensuring that the initial solvent configuration in each window was independent of that in the other windows. The periodic box was orthorhombic, with dimensions of approximately $35 \times 35 \times 51 \AA$ and $37 \times 37 \times 51$ $\AA$ for $\alpha \mathrm{CD}$ and $\beta \mathrm{CD}$ simulations, respectively. The host-guest system was oriented in the simulation box, via noninteracting anchor particles, which allowed the APR process to occur along the long $(z)$ axis of the box; see Henriksen et al. for details. ${ }^{40}$ The parameter/topology file was then hydrogen mass repartitioned (HMR) with AMBER's parmed utility, a process which increases solute hydrogen atom masses to 3.024 Da by transferring mass from adjacent heavy atoms, thereby allowing larger simulation time steps without integration errors or significant changes to the thermodynamics. ${ }^{40,54,55}$ All equilibra- 
Table 2. Summary of Simulation Sets in This Work

\begin{tabular}{|c|c|c|c|c|}
\hline $\begin{array}{l}\text { simulation } \\
\text { set ID }\end{array}$ & host FF & guest FF & solvent & $\begin{array}{c}\text { guest } \\
\text { set }\end{array}$ \\
\hline $\begin{array}{l}\text { Q4RG- } \\
\text { TIP3P }\end{array}$ & Q4MD-CD & $\begin{array}{l}\text { RESP/ } \\
\text { GAFF }\end{array}$ & TIP3P, $\mathrm{Na}^{+}, \mathrm{Cl}^{-}$ & full 43 \\
\hline $\begin{array}{l}\text { Q4RG- } \\
\text { TIP3P- } \\
\text { sm1 }\end{array}$ & Q4MD-CD & $\begin{array}{l}\text { RESP/ } \\
\text { GAFF }\end{array}$ & TIP3P, $\mathrm{Na}^{+}, \mathrm{Cl}^{-}$ & $\begin{array}{c}\text { small } \\
15\end{array}$ \\
\hline $\begin{array}{l}\text { Q4RG- } \\
\text { TIP3P- } \\
\text { sm2 }\end{array}$ & Q4MD-CD & $\begin{array}{l}\text { RESP/ } \\
\text { GAFF }\end{array}$ & TIP3P, $\mathrm{Na}^{+}, \mathrm{Cl}^{-}$ & $\begin{array}{c}\text { small } \\
15\end{array}$ \\
\hline $\begin{array}{l}\text { Q4RG- } \\
\text { TIP3P- } \\
\text { sm3 }\end{array}$ & Q4MD-CD & $\begin{array}{l}\text { RESP/ } \\
\text { GAFF }\end{array}$ & TIP3P, $\mathrm{Na}^{+}, \mathrm{Cl}^{-}$ & $\begin{array}{c}\text { small } \\
15\end{array}$ \\
\hline $\begin{array}{l}\text { Q4RG- } \\
\text { TIP3P-shw }\end{array}$ & Q4MD-CD & $\begin{array}{l}\text { RESP/ } \\
\text { GAFF }\end{array}$ & TIP3P, $\mathrm{Na}^{+}, \mathrm{Cl}^{-}$ & $\begin{array}{c}\text { small } \\
15\end{array}$ \\
\hline $\begin{array}{l}\text { Q4RG- } \\
\text { TIP4Pew }\end{array}$ & Q4MD-CD & $\begin{array}{l}\text { RESP/ } \\
\text { GAFF }\end{array}$ & TIP4Pew, $\mathrm{Na}^{+}, \mathrm{Cl}^{-}$ & full 43 \\
\hline Q4RG-SPC & Q4MD-CD & $\begin{array}{l}\text { RESP/ } \\
\text { GAFF }\end{array}$ & $\mathrm{SPC}, \mathrm{Na}^{+}, \mathrm{Cl}^{-}$ & full 43 \\
\hline Q4RG-OPC & Q4MD-CD & $\begin{array}{l}\text { RESP/ } \\
\text { GAFF }\end{array}$ & $\mathrm{OPC}, \mathrm{Na}^{+}, \mathrm{Cl}^{-}$ & full 43 \\
\hline $\begin{array}{l}\text { Q4RG- } \\
\text { OPC-jc }\end{array}$ & Q4MD-CD & $\begin{array}{l}\text { RESP/ } \\
\text { GAFF }\end{array}$ & $\mathrm{OPC}, \mathrm{Na}^{+}, \mathrm{Cl}^{-}(\mathrm{jc})$ & full 43 \\
\hline $\begin{array}{l}\text { Q4RG- } \\
\text { OPC-phos }\end{array}$ & Q4MD-CD & $\begin{array}{l}\text { RESP/ } \\
\text { GAFF }\end{array}$ & $\begin{array}{l}\mathrm{OPC}, \mathrm{Na}^{+}, \mathrm{H}_{2} \mathrm{PO}_{4}^{-} \text {, } \\
\mathrm{HPO}_{4}^{2-}\end{array}$ & full 43 \\
\hline $\begin{array}{l}\text { Q4RG- } \\
\text { OPC- } \\
\text { jcphos }\end{array}$ & Q4MD-CD & $\begin{array}{l}\text { RESP/ } \\
\text { GAFF }\end{array}$ & $\begin{array}{l}\mathrm{OPC}, \mathrm{Na}^{+}, \mathrm{H}_{2} \mathrm{PO}_{4}^{-}, \\
\mathrm{HPO}_{4}{ }^{2-},(\mathrm{jc})\end{array}$ & full 43 \\
\hline $\begin{array}{l}\text { Q4BG- } \\
\text { TIP3P }\end{array}$ & Q4MD-CD & $\begin{array}{l}\text { AM1-BCC/ } \\
\text { GAFF }\end{array}$ & TIP3P, $\mathrm{Na}^{+}, \mathrm{Cl}^{-}$ & full 43 \\
\hline $\begin{array}{l}\text { BGRG- } \\
\text { TIP3P }\end{array}$ & $\begin{array}{l}\text { AM1-BCC/ } \\
\text { GAFF }\end{array}$ & $\begin{array}{l}\text { RESP/ } \\
\text { GAFF }\end{array}$ & TIP3P, $\mathrm{Na}^{+}, \mathrm{Cl}^{-}$ & full 43 \\
\hline $\begin{array}{l}\text { BGBG- } \\
\text { TIP3P }\end{array}$ & $\begin{array}{l}\text { AM1-BCC/ } \\
\text { GAFF }\end{array}$ & $\begin{array}{l}\text { AM1-BCC/ } \\
\text { GAFF }\end{array}$ & TIP3P, $\mathrm{Na}^{+}, \mathrm{Cl}^{-}$ & full 43 \\
\hline
\end{tabular}

tion and production simulations were carried out with the GPU-capable pmemd.cuda MD engine. Equilibration consisted of 50,000 steps of energy minimization, followed by 100 ps of NVT warming from $0-298.15 \mathrm{~K}$ and $2000 \mathrm{ps}$ of NPT equilibration at $298.15 \mathrm{~K}$. Production simulations were run for a minimum of $5.0 \mathrm{~ns}$ out to a maximum of $50 \mathrm{~ns}$, with the exact length determined by a threshold in the restraint coordinate uncertainty. All simulations used a time step of $4 \mathrm{fs}$, enabled by $\mathrm{HMR}$, with a Langevin thermostat ${ }^{56}$ and a Monte Carlo barostat. $^{57}$ The nonbonded cutoff was set to $9.0 \AA$, and the default AMBER PME parameters (identical for AMBER 14 and 16) were employed.

\section{RESULTS}

We first present a comparative analysis of the various force field combinations tested in this work, focusing on how the choice of parameters affects agreement with experiment, solvent structure, and host conformation. Then we discuss how these factors combine to influence the binding modes of various guests. Finally, in order to provide context for decisions about where to focus force field development efforts, we examine various components of the binding free energy and study how variation in the tested force field parameters propagate into calculated thermodynamic quantities.

Throughout the text, we refer to the specific force field combinations using a "simulation set ID" which indicates the host, guest, and solvent force field, in that order. For example, "Q4RG-OPC" indicates that the Q4MD-CD force field was used for the cyclodextrin host, a RESP/GAFF force field was used for the guest, and the OPC water model and appropriate ion parameters were used for the solvent. Alternatively,
"BGBG-TIP3P" indicates that the AM1-BCC/GAFF force field was used for both the host and guest along with the TIP3P water model and appropriate ions. Table 2 provides a complete summary of the force field combinations tested.

Force Field Comparison and Analysis. Overall Agreement with Experiment. Overall, the force field combinations we tested (Table 2) produced moderate agreement with the experimental reference data from ITC studies; ${ }^{37}$ see Table 3, Tables S1 and S2, Figure 3, and Figure 4. We consider two aspects of agreement with experiment: 1) accuracy, which is reported by metrics such as slope/intercept, RMSE, MSE, and MUE, and 2) ranking ability, as reported by the coefficient of determination $\left(R^{2}\right)$ and Kendall's rank correlation coefficient $(\tau)$. The binding free energy and enthalpy calculations had an RMSE range of $0.9-1.8 \mathrm{kcal} / \mathrm{mol}$ and $0.9-4.0 \mathrm{kcal} / \mathrm{mol}$, respectively. No single force field combination emerged as superior to the others at computing both binding free energy and enthalpy. The Q4RG-TIP3P force field, which we expect would be the initial, "default" choice for an AMBER user, shows poor correlation $\left(R^{2}=0.47\right)$ and significant deviation $(\mathrm{RMSE}=$ $1.8 \mathrm{kcal} / \mathrm{mol}$ ) from experimental binding free energies, making it one of the worst force fields tested. For binding enthalpies, the Q4RG-TIP3P force field showed improved correlation $\left(R^{2}\right.$ $=0.68)$ but even greater deviation from experiment $(\mathrm{RMSE}=$ $2.0 \mathrm{kcal} / \mathrm{mol}$ ). Surprisingly, the best force field for calculating the binding free energy was the BGBG-TIP3P combination, which produced the highest correlation $\left(R^{2}=0.60\right)$ and lowest deviation $(\mathrm{RMSE}=0.9 \mathrm{kcal} / \mathrm{mol})$ to experimental values of all force fields tested. The BGBG-TIP3P combination was expected to perform poorly because we crudely parametrized the CD host molecule and made no effort to tune its experimentally known conformational properties, as was done for the Q4MD-CD force field. ${ }^{46}$ On the other hand, the BGBGTIP3P combination was among the worst at calculating the binding enthalpy, showing low correlation $\left(R^{2}=0.39\right)$ and high deviation $(\mathrm{RMSE}=2.6 \mathrm{kcal} / \mathrm{mol}$ ) relative to experiment. The best force field for binding enthalpies, Q4RG-TIP4Pew $\left(R^{2}=\right.$ $0.77, \mathrm{RMSE}=0.9 \mathrm{kcal} / \mathrm{mol})$, produced mediocre binding free energy results, similar to those observed for Q4RG-TIP3P.

Interestingly, when each class of guest molecule is considered separately, the computed binding free energies correlate better with experiment, relative to the correlation of the entire data set. For example, in the Q4RG-TIP3P data set, the $R^{2}$ values for ammoniums, cyclic alcohols, and carboxylates are 0.95, 0.84, and 0.83 , respectively (Figure 3 , top and Table S3), which greatly improves on the 0.47 overall value. However, since each group is shifted relative to one another, the accuracy successively diminishes for each group (RMSE $=0.6,1.3$, and $2.5 \mathrm{kcal} / \mathrm{mol}$, respectively). The same trend is not generally observed for the binding enthalpy, where only the ammonium guests have significantly higher correlation $\left(R^{2}=0.89\right)$ than the full data set. Similar behavior is observed for other force field combinations. Note that the statistical metrics reported here were generated using resampling with replacement, to provide uncertainty values that account for experimental uncertainty, numerical uncertainty, and differences in data set size. The uncertainties for the $R^{2}$ values are provided in Figure 3 and Figure 4 and Table S3.

The full data set can be subgrouped in a variety of different ways. Error metrics for the following subgroupings are provided in Table S3: ammonium guests, alcohol guests, carboxylate guests, $\alpha \mathrm{CD}$ hosts, $\beta \mathrm{CD}$ hosts, $n$-alkyl guests, aliphatic carboxylate guests, and aromatic carboxylate guests. Some 


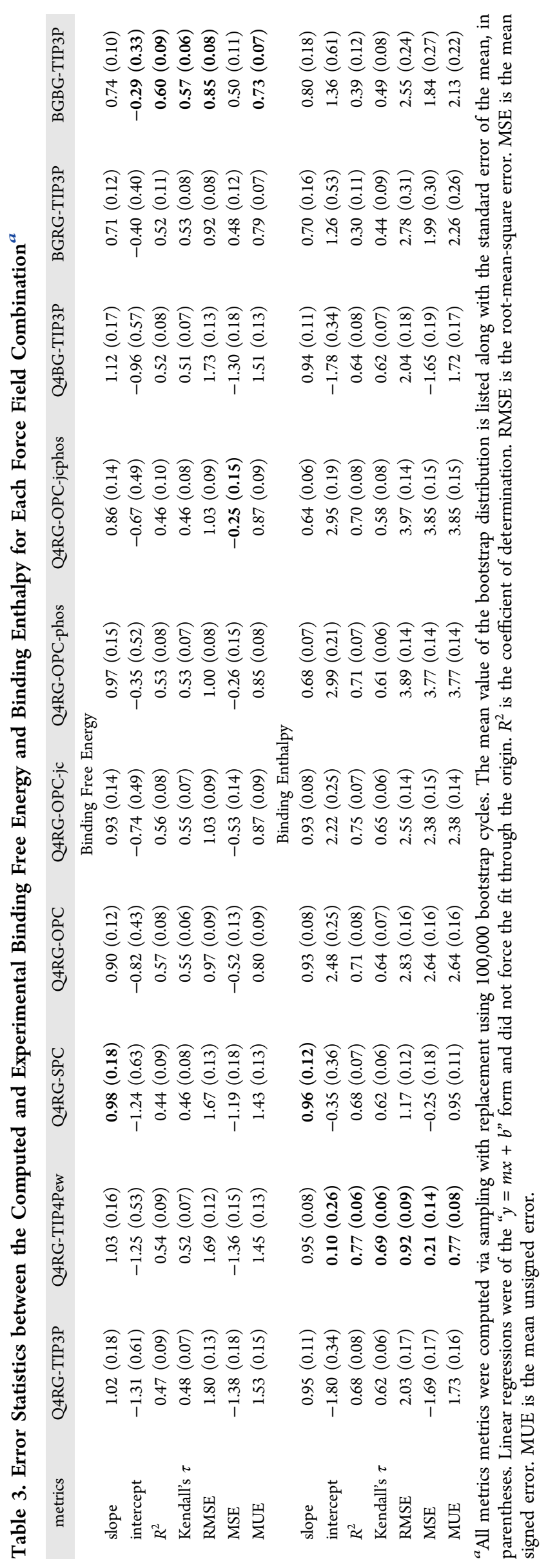



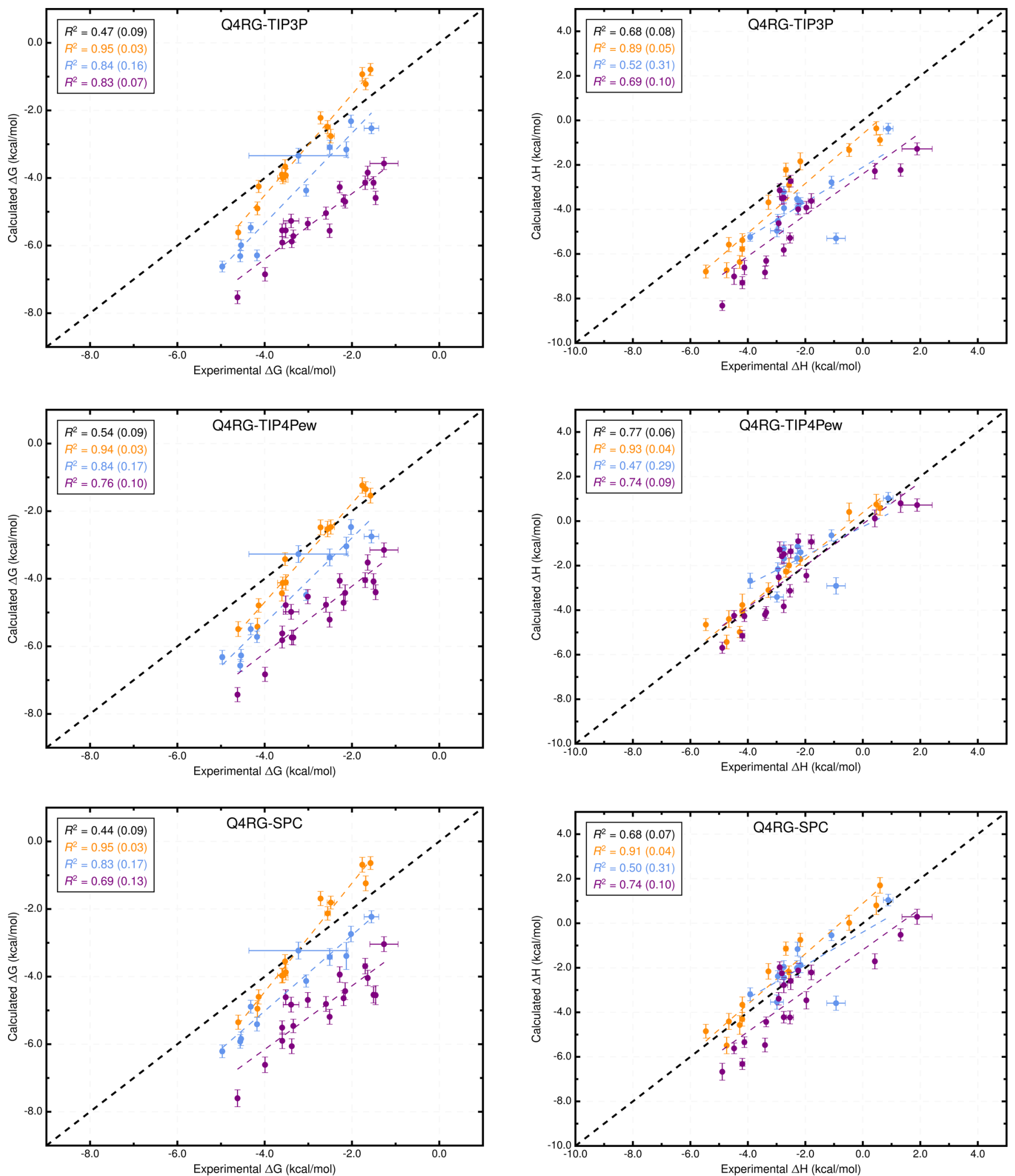

Figure 3. Comparison of calculated binding free energies and binding enthalpies with experiment: (top row) Q4RG-TIP3P, (middle row) Q4RGTIP4Pew, and (bottom row) Q4RG-SPC. The orange, blue, and purple colors distinguish the functional group of the guest as an ammonium, alcohol, or carboxylate, respectively. The overall $R^{2}$ is indicated in black followed by values for each guest functional group colored as mentioned previously.

interesting observations can be made from this data. For example, comparison of the latter two groups, aliphatic and aromatic carboxylate guests, shows that although their binding free energy RMSE is similar, the correlation with experiment is much higher for the aliphatic carboxylates than the aromatic across all force field combinations. This same trend is even more pronounced for the binding enthalpy, although the RMSE differences are a bit larger. Another example involves comparing binding to the $\alpha \mathrm{CD}$ host versus the $\beta \mathrm{CD}$ host. For most of the force field combinations which use the Q4MD- 



Figure 4. Comparison of calculated binding free energies and binding enthalpies with experiment: (top row) Q4RG-OPC, (middle row) Q4RG-OPCphos, and (bottom row) BGBG-TIP3P. The orange, blue, and purple colors distinguish the functional group of the guest as an ammonium, alcohol, or carboxylate, respectively. The overall $R^{2}$ is indicated in black followed by values for each guest functional group colored as mentioned previously.

CD force field on the host, the RMSE values are similar between the two groups and the correlation is marginally better for the $\beta C D$ group. In contrast, for the BGBG-TIP3P force field combination, the correlation is significantly higher for $\alpha \mathrm{CD}$ group than the $\beta \mathrm{CD}$ group for the binding free energy and enthalpy.

The entropic and enthalpic components of the binding free energy provide an additional perspective for comparison with experiment. Although we did not compute the entropic 
Table 4. Mean Enthalpic and Entropic Components of Binding Free Energy, for Each Class of Host-Guest Pair, and Their Standard Deviation, in Parentheses, Across All Guests in Each Class ( $\mathrm{kcal} / \mathrm{mol})^{a}$

\begin{tabular}{|c|c|c|c|c|c|c|c|c|c|c|c|c|c|}
\hline \multirow{2}{*}{$\begin{array}{l}\text { Host } \\
\text { Type }\end{array}$} & \multirow{2}{*}{ Guest Type } & \multicolumn{2}{|c|}{ Experiment } & \multicolumn{2}{|c|}{ Q4RG-TIP3P } & \multicolumn{2}{|c|}{ Q4RG-TIP4Pew } & \multicolumn{2}{|c|}{ Q4RG-SPC } & \multicolumn{2}{|c|}{ Q4RG-OPC } & \multicolumn{2}{|c|}{ BGBG-TIP3P } \\
\hline & & $\Delta \mathrm{H}$ & $-\mathrm{T} \Delta \mathrm{S}$ & $\Delta \mathrm{H}$ & $-\mathrm{T} \Delta \mathrm{S}$ & $\Delta \mathrm{H}$ & $-T \Delta S$ & $\Delta \mathrm{H}$ & $-\mathrm{T} \Delta \mathrm{S}$ & $\Delta \mathrm{H}$ & $-\mathrm{T} \Delta \mathrm{S}$ & $\Delta \mathrm{H}$ & $-\mathrm{T} \Delta \mathrm{S}$ \\
\hline$\alpha C D$ & $\begin{array}{l}\text { Aliphatic } \\
\text { Ammoniums }\end{array}$ & $\begin{array}{l}-3.8 \\
(1.1)\end{array}$ & $\begin{array}{c}0.7 \\
(0.1)\end{array}$ & $\begin{array}{l}-4.7 \\
(1.9)\end{array}$ & $\begin{array}{c}1.6 \\
(0.4)\end{array}$ & $\begin{array}{l}-3.6 \\
(1.3)\end{array}$ & $\begin{array}{c}0.2 \\
(0.6)\end{array}$ & $\begin{array}{l}-3.4 \\
(1.7)\end{array}$ & $\begin{array}{c}0.3 \\
(0.4)\end{array}$ & $\begin{array}{l}-1.7 \\
(1.0)\end{array}$ & $\begin{array}{l}-1.1 \\
(0.8)\end{array}$ & $\begin{array}{l}-1.9 \\
(1.3)\end{array}$ & $\begin{array}{l}-0.5 \\
(0.3)\end{array}$ \\
\hline$\alpha C D$ & $\begin{array}{l}\text { Cyclic } \\
\text { Alcohols }\end{array}$ & $\begin{array}{l}-2.4 \\
(1.0)\end{array}$ & $\begin{array}{l}-0.1 \\
(1.5)\end{array}$ & $\begin{array}{l}-4.4 \\
(1.0)\end{array}$ & $\begin{array}{c}1.4 \\
(0.6)\end{array}$ & $\begin{array}{l}-2.3 \\
(1.0)\end{array}$ & $\begin{array}{l}-0.7 \\
(0.7)\end{array}$ & $\begin{array}{l}-2.9 \\
(0.8)\end{array}$ & $\begin{array}{l}-0.3 \\
(0.6)\end{array}$ & $\begin{array}{l}-0.5 \\
(0.3)\end{array}$ & $\begin{array}{l}-2.2 \\
(0.1)\end{array}$ & $\begin{array}{l}-1.8 \\
(1.0)\end{array}$ & $\begin{array}{c}0.3 \\
(0.7)\end{array}$ \\
\hline$\alpha C D$ & $\begin{array}{l}\text { Aliphatic } \\
\text { Carboxylates }\end{array}$ & $\begin{array}{l}-3.7 \\
(0.8)\end{array}$ & $\begin{array}{c}0.5 \\
(0.4)\end{array}$ & $\begin{array}{l}-6.7 \\
(0.9)\end{array}$ & $\begin{array}{c}0.9 \\
(0.2)\end{array}$ & $\begin{array}{l}-4.3 \\
(0.8)\end{array}$ & $\begin{array}{l}-1.3 \\
(0.5)\end{array}$ & $\begin{array}{l}-5.3 \\
(0.9)\end{array}$ & $\begin{array}{l}-0.4 \\
(0.3)\end{array}$ & $\begin{array}{l}-1.5 \\
(0.5)\end{array}$ & $\begin{array}{l}-3.0 \\
(0.8)\end{array}$ & $\begin{array}{l}-2.9 \\
(1.2)\end{array}$ & $\begin{array}{c}0.1 \\
(0.4)\end{array}$ \\
\hline$\beta C D$ & $\begin{array}{l}\text { Aliphatic } \\
\text { Ammoniums }\end{array}$ & $\begin{array}{c}0.2 \\
(0.6)\end{array}$ & $\begin{array}{l}-3.1 \\
(0.0)\end{array}$ & $\begin{array}{l}-0.9 \\
(0.5)\end{array}$ & $\begin{array}{l}-2.2 \\
(0.4)\end{array}$ & $\begin{array}{c}0.6 \\
(0.2)\end{array}$ & $\begin{array}{l}-3.6 \\
(0.8)\end{array}$ & $\begin{array}{c}0.8 \\
(0.8)\end{array}$ & $\begin{array}{l}-3.5 \\
(0.5)\end{array}$ & $\begin{array}{c}2.9 \\
(0.3)\end{array}$ & $\begin{array}{l}-5.2 \\
(0.9)\end{array}$ & $\begin{array}{c}2.5 \\
(0.2)\end{array}$ & $\begin{array}{l}-4.5 \\
(0.8)\end{array}$ \\
\hline$\beta C D$ & $\begin{array}{l}\text { Cyclic } \\
\text { Alcohols }\end{array}$ & $\begin{array}{l}-2.0 \\
(1.5)\end{array}$ & $\begin{array}{l}-1.9 \\
(0.5)\end{array}$ & $\begin{array}{l}-3.4 \\
(1.6)\end{array}$ & $\begin{array}{l}-1.9 \\
(0.5)\end{array}$ & $\begin{array}{l}-1.2 \\
(1.2)\end{array}$ & $\begin{array}{l}-4.1 \\
(0.4)\end{array}$ & $\begin{array}{l}-1.4 \\
(1.4)\end{array}$ & $\begin{array}{l}-3.5 \\
(0.3)\end{array}$ & $\begin{array}{c}1.3 \\
(0.7)\end{array}$ & $\begin{array}{l}-5.7 \\
(0.8)\end{array}$ & $\begin{array}{c}1.9 \\
(1.3)\end{array}$ & $\begin{array}{l}-4.8 \\
(1.6)\end{array}$ \\
\hline$\beta C D$ & $\begin{array}{l}\text { Aliphatic } \\
\text { Carboxylates }\end{array}$ & $\begin{array}{c}1.2 \\
(0.7)\end{array}$ & $\begin{array}{l}-3.5 \\
(0.3)\end{array}$ & $\begin{array}{l}-1.9 \\
(0.6)\end{array}$ & $\begin{array}{l}-2.4 \\
(0.5)\end{array}$ & $\begin{array}{c}0.5 \\
(0.4)\end{array}$ & $\begin{array}{l}-4.6 \\
(0.7)\end{array}$ & $\begin{array}{l}-0.6 \\
(1.0)\end{array}$ & $\begin{array}{l}-3.3 \\
(0.2)\end{array}$ & $\begin{array}{c}3.6 \\
(0.1)\end{array}$ & $\begin{array}{l}-6.7 \\
(1.0)\end{array}$ & $\begin{array}{c}0.9 \\
(1.3)\end{array}$ & $\begin{array}{l}-3.4 \\
(2.1)\end{array}$ \\
\hline$\beta C D$ & $\begin{array}{l}\text { Aromatic } \\
\text { Carboxylates }\end{array}$ & $\begin{array}{l}-2.5 \\
(0.4)\end{array}$ & $\begin{array}{c}0.1 \\
(0.9)\end{array}$ & $\begin{array}{l}-3.6 \\
(0.6)\end{array}$ & $\begin{array}{l}-1.2 \\
(0.6)\end{array}$ & $\begin{array}{l}-1.6 \\
(0.6)\end{array}$ & $\begin{array}{l}-3.0 \\
(0.8)\end{array}$ & $\begin{array}{l}-2.6 \\
(0.6)\end{array}$ & $\begin{array}{l}-2.0 \\
(0.7)\end{array}$ & $\begin{array}{c}1.2 \\
(0.9)\end{array}$ & $\begin{array}{l}-4.8 \\
(1.2)\end{array}$ & $\begin{array}{l}-0.2 \\
(1.2)\end{array}$ & $\begin{array}{l}-2.4 \\
(1.2)\end{array}$ \\
\hline
\end{tabular}

${ }^{a}$ The orange color indicates a negative, favorable value; the blue color indicates a positive, unfavorable value.
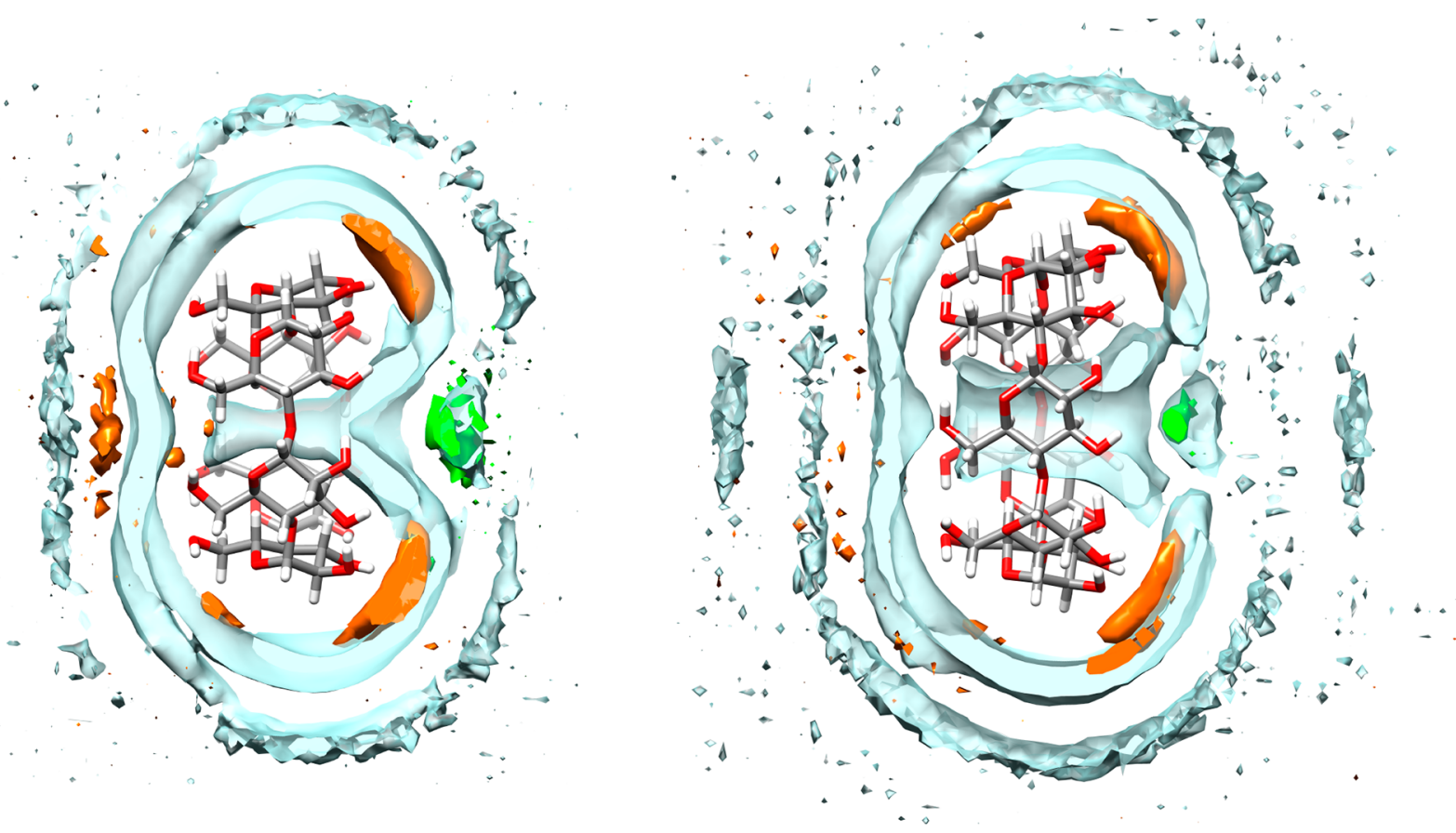

Figure 5. Density contour plots for water (clear blue), sodium ions (orange), and chloride ions (green) around $\alpha \mathrm{CD}$ (left) and $\beta \mathrm{CD}$ (right). The water and ion contour levels are 1.05 and 2.00 times bulk density, respectively. For clarity, only a $2.5 \AA$ thick surface slice is shown for each solvent component.

component directly, we obtain it from subtraction of the binding enthalpy from the binding free energy, as done in ITC experiments. From the experimental data, it was observed that some degree of entropy-enthalpy compensation occurs when comparing binding between the smaller $\alpha \mathrm{CD}$ and larger $\beta \mathrm{CD}{ }^{37}$ For the present guests, the binding enthalpy to $\alpha \mathrm{CD}$ is generally more favorable than binding to $\beta \mathrm{CD}$, likely reflecting stronger dispersion interactions generated by a snug fit. In contrast, the entropic contribution to the binding free energy is more favorable moving from $\alpha \mathrm{CD}$ to $\beta \mathrm{CD}$, presumably due to greater conformational freedom of the guest and the ejection of additional ordered water from the larger host. These trends are also observed in our computational values, although the magnitude of the thermodynamic components varies widely across different force field combinations (Table 4). The trend is even observed for the BGBG-TIP3P force field which, as discussed below, explores significantly different conformations than the Q4RG force field.

Comparison of Water Models, Ion Parameters, and Buffer Components. Changing the water model is one of the obvious force field comparisons to make in AMBER, since most AMBER solute force fields are not parametrized against a specific water model. In combination with the Q4MD-CD host force field (Q4) and the RESP/GAFF guest force field (RG), we evaluated four water models: TIP3P, ${ }^{18}$ TIP4Pew, ${ }^{19}$ SPC/ $\mathrm{E}^{20}$ and $\mathrm{OPC}^{21}$ (Table 2). For binding free energy, the error metrics with respect to experiment are uniformly mediocre for the traditional water models (Q4RG-TIP3P, Q4RG-TIP4Pew, and Q4RG-SPC in Table 3 and Figure 3). Thus, the correlation was low $\left(R^{2}=0.44-0.54\right)$, and the deviation from experiment was significant $(\mathrm{RMSE}=1.7-1.8 \mathrm{kcal} / \mathrm{mol})$. In general, these water models overestimate the binding affinity, particularly for 
carboxylate guests, leading to an MSE of $-1.2 \mathrm{kcal} / \mathrm{mol}$ or worse. Relative to the four more traditional water models, the Q4RG-OPC combination showed improvement in every error metric category except slope, including higher correlation $\left(R^{2}=\right.$ $0.57)$ and much lower deviation from experiment $(\mathrm{RMSE}=1.0$ $\mathrm{kcal} / \mathrm{mol}$ ).

More variation is observed for the binding enthalpy calculations (Table 3, Figure 3). As noted previously, Q4RGTIP4Pew performed the best relative to experiment $\left(R^{2}=0.77\right.$, RMSE $=0.9 \mathrm{kcal} / \mathrm{mol}$ ), and Q4RG-SPC results were only slightly worse $\left(R^{2}=0.68, \mathrm{RMSE}=1.2 \mathrm{kcal} / \mathrm{mol}\right)$. The Q4RGTIP3P force field significantly overestimates the binding enthalpy (MSE $=-1.7 \mathrm{kcal} / \mathrm{mol}$ ), while the Q4RG-OPC force field underestimates by even more $(\mathrm{MSE}=2.8 \mathrm{kcal} / \mathrm{mol})$. The relative MSE trend observed here between Q4RG-TIP3P and QR4G-TIP4Pew is opposite to that found for the CB7 host where the binding enthalpy was overestimated significantly more by TIP4Pew than TIP3P. ${ }^{58}$ The increase in correlation with experiment for individual guest classes over the entire data set is not as noticeable for the binding enthalpy, although for ammonium guests the $R^{2}$ value equals or exceeds 0.85 for all four force field combinations.

We next considered whether the structured water in the $C D$ cavity differs significantly between water models. To evaluate this, we computed the water and sodium ion radial distribution functions (RDF) referenced to the center of mass of the unbound CD (Figure S11). For the simulation sets which used the Q4MD-CD force field, the RDFs are quite similar, suggesting that the water structure in the $\mathrm{CD}$ cavity does not change across the four water models we tested. Indeed, regions of solvent density around the empty CD host share the same features, differing only in the relative magnitude, and reveal a core of structured water within the CD cavity and ion binding regions near the hydroxyl group on the exterior of the $\mathrm{CD}$ host (e.g., Q4RG-TIP3P, Figure 5). The Q4RG-TIP4Pew and Q4RG-SPC force field combinations show a large peak in the sodium RDFs at 7-8 $\AA$, which is not observed to the same degree for the other two solvent models. This appears to correspond with sodium binding between the $\mathrm{O} 2$ and $\mathrm{O} 3$ hydroxyls at the secondary face of the $\mathrm{CD}$. Comparison of the density grids across all four solvent models suggests that the high-density water and sodium regions occupy the same locations but vary in magnitude.

Although the ITC studies which generated the comparison data for this study used $50 \mathrm{mM}$ sodium phosphate buffer, we chose to use equivalent ionic strength sodium chloride for most force field combinations, due to the lack of published parameters for mono- and divalent phosphate. However, we wanted to test whether phosphate buffer influenced the binding calculations, even though it is not expected to interact significantly with CD hosts. The OPC model was the only water model tested for which we did not observe unphysical aggregation of phosphate buffer ions during simulation. Therefore, we performed several more simulation sets which evaluated how changes to sodium chloride parameters or inclusion of phosphate affect the results. The Q4RG-OPC simulation set used $\mathrm{Na}^{+}$and $\mathrm{Cl}^{-}$parameters which were tuned to reproduce hydration free energies in OPC water. However, as these were not available until some time after publication of the OPC water model, several research groups have used the Joung-Cheatham parameters tuned for TIP4Pew. ${ }^{59,60}$ The OPC-tuned parameters $\left(R_{\min / 2}=1.432,2.298 \AA, \varepsilon=0.0215\right.$, $0.6366 \mathrm{kcal} / \mathrm{mol}$ for $\mathrm{Na}^{+}$and $\mathrm{Cl}^{-}$, respectively) differ substantially (Figure S18) from the Joung-Cheatham parameters $\left(R_{\min / 2}=1.226,2.760 \AA, \varepsilon=0.1684,0.0117 \mathrm{kcal} / \mathrm{mol}\right.$ for $\mathrm{Na}^{+}$and $\mathrm{Cl}^{-}$, respectively), so we first evaluated these parameters. The Q4RG-OPC-jc simulation set is identical to the Q4RG-OPC set, with the exception of these ion parameters, and the results show very little difference in terms of absolute calculated value (Tables S8 and S9) or agreement with experiment (Table 3). We then evaluated whether inclusion of phosphate buffer at $50 \mathrm{mM}$, to exactly match experimental conditions, rather than $\mathrm{NaCl}$ would affect the results. We tested the phosphate buffer with both the OPCtuned (Q4RG-OPC-phos) and Joung-Cheatham (Q4RG-OPCjcphos) parameters for sodium. Relative to the OPC simulation sets with just $\mathrm{NaCl}$ buffer, the free energy calculations with phosphate buffer yield comparable error statistics with experiment (Table 3 and Figure 4). In contrast, the binding enthalpy results show a further positive shift of between 1.1 and $1.4 \mathrm{kcal} / \mathrm{mol}$ in MSE relative to the OPC $/ \mathrm{NaCl}$ buffer, likely due to a phosphate ion binding near the $\mathrm{CD}$ cavity. The slope of the linear regression between calculated and experiment also changes from approximately 0.9 to 0.65 when switching from $\mathrm{NaCl}$ buffer to phosphate.

Impact of the Host Force Field. Most of the force field combinations studied use Q4MD-CD ${ }^{46}$ for the CD host molecules. This force field was designed to agree with experimentally determined conformational properties of CDs, mostly from NMR data. We were interested in how this particular choice of force field might impact binding calculations. To provide a "control", we created a CD force field using a minimal effort approach which essentially involved parametrizing the glucose monomer with AM1-BCC charges and the GAFF force field (Methods). The Lennard-Jones parameters for the two approaches are identical, and the charges are very similar (Figure S13), with maximum deviations of 0.26 or 0.13 on the $\mathrm{C} 1$ and $\mathrm{O} 1$ atoms, respectively. The primary difference between the two force fields is the dihedral parameters. The Q4MD-CD force field primarily draws its parameters from the GLYCAM04 force field, ${ }^{47-49}$ which is optimized for carbohydrates, except that the 1-4 electrostatic and 1-4 nonbonded interaction are set to 1.2 and 2.0, respectively, rather than unity, to be consistent with other major AMBER force fields.

Simulations of unbound $\alpha \mathrm{CD}$ and $\beta \mathrm{CD}$ show that the conformational distribution is significantly different between these two force fields. The population histograms for the "flip" pseudotorsion, which reports the orientation of a glucose monomer relative to its neighbor, show that $C D$ hosts in the BGBG-TIP3P simulation set have several highly populated flip orientations that are not observed in the Q4RG-TIP3P simulations (Figure S14A, B). These alternate conformations collapse the cavity of the $\mathrm{CD}$ molecule and prevent water from locating there (Figure S11, top). The guest binding mode appears to be less structured for BGBG-TIP3P as well. This is particularly noticeable for some of the smaller guests, such as cyclopentanol, binding to $\beta \mathrm{CD}$ (Figure S15). An overlay of distance histograms for each bound guest relative to the $\mathrm{CD}$ center of mass shows much more dispersed and bimodal distributions for BGBG-TIP3P than for Q4RG-TIP3P (Figure S16A,B). For some of the host-guest pairs with dispersed distance histograms, particularly the cycloalcohols, the binding was so weak or unstable that the "wall restraints" may have influenced calculated results (see Methods). In spite of what appears to be a crude force field representation, which allows 
excessive conformational flexibility to the host, the BGBGTIP3P binding free energies give the highest correlation $\left(R_{2}=\right.$ $0.60)$ and lowest RMSE $(0.9 \mathrm{kcal} / \mathrm{mol})$ to experiment of all the simulation sets tested (Table 3). On the other hand, the binding enthalpy results are poor: the correlation is poor $\left(R^{2}=\right.$ $0.39)$ and the RMSE $(2.6 \mathrm{kcal} / \mathrm{mol})$ is only exceeded by the simulation sets using the OPC water model, which shifts the enthalpy in the positive direction (Table 3). Further comparison of these two host force fields is given in the "Binding Modes" section.

Comparison of the RESP and AM1-BCC Charge Models. Two common methods for partial charge assignment in AMBER are the RESP ${ }^{53,61}$ and AM1-BCC ${ }^{50,51}$ approaches. The latter approximates the former for a fraction of the computational cost and is implemented in and widely used via AMBER's antechamber program. We assigned guest partial charges with both methods and noticed substantial differences for certain atom types (Figure S12), particularly the ammonium nitrogen (atom type nh) and certain aliphatic carbon atoms (c3) between aromatic groups and carboxylates. (However, compensatory changes in neighboring atom partial charges may mitigate these differences in terms of the electrostatic field generated by the entire guest molecule.) To evaluate the degree to which these differences affect the binding results, we performed a new set of simulations, Q4BG-TIP3P, keeping all parameters identical to Q4RG-TIP3P except the guest charges, which were generated with AM1-BCC instead of RESP. In terms of agreement with experiment, the error metrics for Q4BG-TIP3P are indistinguishable from Q4RG-TIP3P for both binding free energy and enthalpy (Table 3). Additionally, for both free energy and enthalpy, the correlation between the two simulation sets is high $\left(R^{2}>0.92\right)$, and the slope and intercept for linear regression between the data sets are approximately 0.99 and 0.01 , respectively. We conclude that guest partial charge assignment with the RESP and AM1-BCC method yields equivalent results for the host-guest pairs study in this work.

We furthermore performed binding calculations using the AM1-BCC/GAFF force field for the CD hosts and the RESP/ GAFF force field for the guests, referred to as the BGRGTIP3P simulation set. As anticipated, using mixed approaches to generate partial atomic charges - RESP for the guests and AM1-BCC for the hosts - also had little impact on the error with experiment (Table 3, compare BGRG-TIP3P and BGBGTIP3P) or the thermodynamic decomposition (compare Figure S8 with Figure S9 and Table S13 with Table S14).

Binding Modes. The reported binding values in Table S1 are obtained from two separate calculations corresponding to the polar group of the guest lying at either the primary or secondary face of the asymmetric cavity. Inspection of the binding free energy values for each individual binding orientation is informative (Tables S4-S14). One interesting result is that, for all force field combinations using the Q4MD$\mathrm{CD}$ host force field, the ammonium guests prefer, by about 0.5-2.0 $\mathrm{kcal} / \mathrm{mol}$, orienting their polar group toward the primary face, which is not expected, ${ }^{37,62}$ particularly as one might anticipate that the cationic ammonium group could be better hydrated at the larger secondary face. With the exception of 1-methylcyclohexanol binding to $\beta \mathrm{CD}$ (b-mch), in which the nonpolar methyl group likely competes with hydroxyl group for control of the orientation, all of the carboxylate and alcohol guests prefer to orient their polar group toward the secondary face of CD. In contrast, the BGBG-TIP3P and BGRG-TIP3P force field combinations display equivalent affinity between orientations or slightly favor the secondary orientation for all guest classes.

Solvent Structure. The solvent density distribution near the binding cavity of free $\alpha \mathrm{CD}$ and $\beta \mathrm{CD}$ indicates a possible explanation for the observed orientational preferences observed when using the Q4MD-CD force field for the hosts (Figure 5). The secondary face of the CD features a high density chloride binding region at the center of the opening, surrounded by several high density sodium binding zones near the secondary alcohols. In contrast, the primary side of the $\mathrm{CD}$ only has a moderate affinity sodium binding zone near the center of the cavity opening. Thus, it appears that the CD structure itself naturally encodes some degree of preference for placing positively charged groups at the primary face and negatively charged groups at the secondary face, at least for the Q4MD$\mathrm{CD}$ force field. In contrast, the "BG" host force field produces such a flexible, collapsed unbound host structure that welldefined localization of solvent density is not observed (data not shown).

Entropic Considerations. The entropic component of binding is strongly linked to the binding orientation. The entropic contribution from the secondary orientation is more favorable than that for the primary orientation for all guests in the Q4RG-TIP3P data set, except 1-methylcyclohexanol, cycloheptanol, and cyclooctanol (Table S4 and Figure 7, bottom). The unusual binding mode for these guests, as discussed below, likely accounts for deviation in the trend. One possible explanation for the relative entropic favorability of the secondary orientation lies in the interaction of the $C D$ hydroxyls with the polar headgroup of the guest. When the guest is in the primary orientation, the polar headgroup lies nearer to all the primary face hydroxyls than when it is in the secondary orientation. This may allow it to act as a clamp, perhaps via hydrogen bonding and/or electrostatic interactions, limiting the motion of the $\mathrm{CD}$. We investigated this possibility by computing the root mean squared fluctuation (RMSF) value for all CD oxygen atoms, both in the bound and unbound state. By summing the RMSF difference between the bound and unbound states we estimated a crude measure of CD flexibility for a selection $n$-alkyl guests binding to $\alpha \mathrm{CD}$ (Figure S3, top). The difference in CD fluctuations before and after binding is clearly much larger when the guest binds in the primary orientation rather the secondary, which matches the entropic results (Figure 7, bottom). However, the increase in the RMSF difference of the secondary orientation as the alkyl chain gets longer is not reflected in the entropy results which suggests an additional mechanism is at play, possibly related to structured water.

Enthalpic Considerations. The binding enthalpy for both orientations of the guest can be further broken down into component force field terms, namely the valence energy terms (bonds, angles, torsions, and 1-4 interactions), and the nonbonded Lennard-Jones (LJ) and electrostatic terms (Table S4). Focusing on a selected set of $n$-alkyl ammonium and carboxylate guests, we observe that the uniformly favorable binding enthalpy in the primary orientation is driven by the valence and LJ terms and opposed by the electrostatic term (Figure 7, top). Binding in the secondary orientation is more complex. The profile for ammonium guests is similar to what is observed for the primary orientation, although the magnitude of each term is generally smaller (Figure 7, center). For the carboxylate guests, however, the valence terms are negligible, 

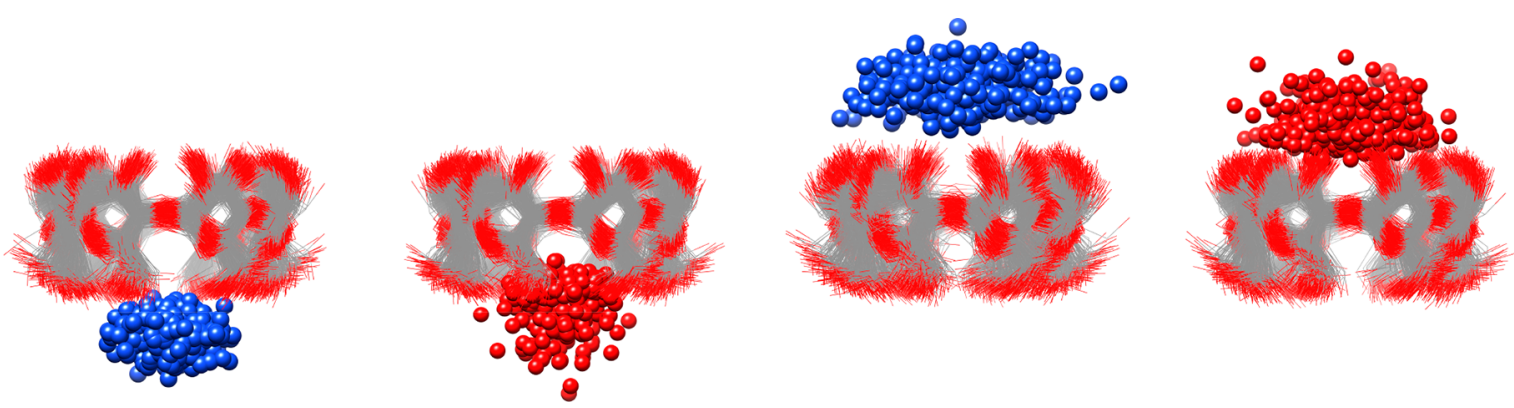

Figure 6. Overlay of 400 simulation snapshots, spaced $2.5 \mathrm{~ns}$ apart, showing the position of a carboxylate oxygen atom (red spheres) and an ammonium nitrogen atom (blue spheres) relative to the $\alpha \mathrm{CD}$ host when bound in the primary orientation (left side) and secondary orientation (right side). Snapshots were taken from trajectories of the a-ham and a-hep host-guest pairs in the Q4RG-TIP3P simulation set.
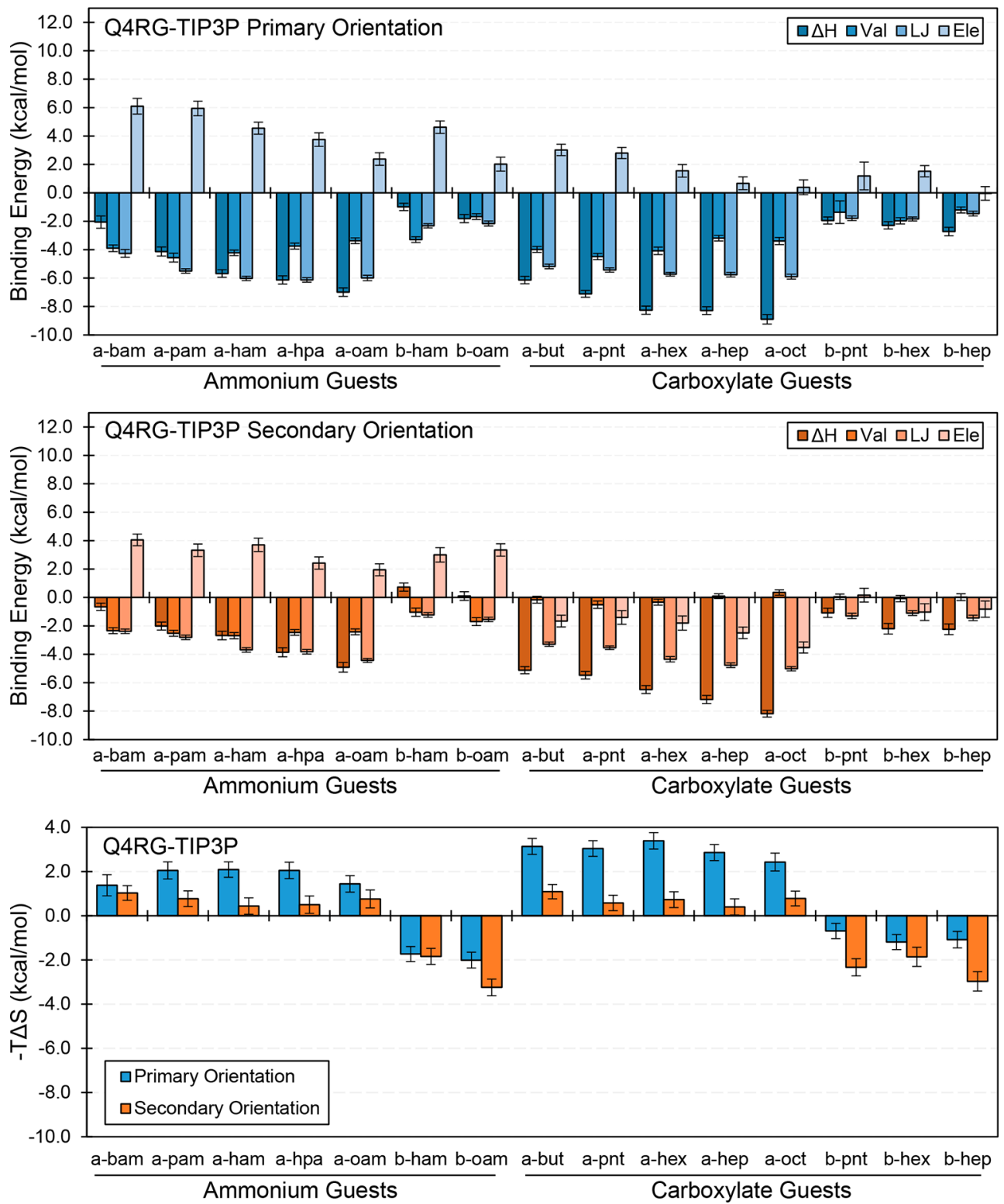

Figure 7. Binding enthalpy decompositions for the primary orientation (top/blue) and secondary orientation (center/orange) and entropic contribution to the free energy for both orientations (bottom) for selected guests from the Q4RG-TIP3P simulation set. The number of guest carbon atoms increases from left to right for each guest class (see Table 1 for identification). Val = valence energy, LJ = Lennard-Jones energy, Ele $=$ electrostatic energy.

whereas the electrostatic term joins the LJ term as a favorable contributor. The branched and unsaturated ammonium and carboxylate guests not shown in Figure 7 follow a similar pattern. 
The cyclic alcohol guests are generally characterized by favorable valence and VDW terms along with an unfavorable electrostatic contribution (Table S4). Two exceptions to this trend are found for cycloheptanol and cyclooctanol binding to $\alpha \mathrm{CD}$. For these cases, in which the guests' size prevents a complete fit into the host cavity, the valence energy term is unfavorable in the primary orientation, and the electrostatic energy is favorable for both orientations. The aromatic carboxylate guests bind with a variety of enthalpic profiles although all have favorable LJ contributions - which likely reflects the various placement of substituents on the aromatic ring.

In contrast to what is observed for the Q4MD-CD force fields, the decomposed enthalpy for the "BG" force fields reveals a completely different thermodynamic profile (Table S14 and Figure S9). First, the profile for binding in either the primary and secondary orientation is very similar (Figure S9, top and middle). Additionally, the valence term is almost always unfavorable and is driven specifically by the dihedral component which can be as large as $+10-12 \mathrm{kcal} / \mathrm{mol}$ (Table S15). Finally, the LJ and electrostatic terms are large and negative for most host-guest pairs.

Structural Interpretation of the Thermodynamic Components of Binding. Here, we investigate structural explanations for the observed thermodynamic values, primarily focusing on the Q4RG-TIP3P data set.

We focus first on the valence energy term, which we consider to comprise the bond, angle, torsion, and 1-4 electrostatic and 1-4 LJ energy terms. (The 1-4 term is included because it only occurs intramolecularly, between atoms which are tethered to each other by three bonds.) The 1-4 electrostatic term is responsible for the observation that carboxylates bind with favorable valence energy in the primary orientation but not the secondary orientation (Table S15). It appears to play a key role in the magnitude of the bonded term for the other guests as well. Inspection of the $\mathrm{CD}$ dihedral angles suggested that the $\mathrm{O} 6$ and $\mathrm{O} 2$ hydroxyl torsions likely contribute the most to this energy term. Thus, the population histograms for these torsions in the bound state reveal unique profiles, which are dependent both on the guest functional group (ammonium vs carboxylate) and binding orientation (primary vs secondary). Figure S2 demonstrates that, relative to the unbound state (dashed lines), the four guest binding scenarios considered presently (ammonium/primary, ammonium/secondary, carboxylate/primary, carboxylate/secondary) generate unique torsion populations in the $\mathrm{O} 6$ and $\mathrm{O} 2$ hydroxyl groups of the host CD.

The LJ term, favorable in all cases, can be rationalized by considering the greater ability of the guests to pack into the CD cavity relative to water, much as previously seen for guests binding the cucurbituril CB7. ${ }^{58}$ As expected, this term moderately scales with the size of the guest, reflecting the greater number of atoms packed into the cavity. Additionally, the magnitude of the LJ term is largest for the guest in the primary orientation, where interactions with the narrower end of the CD cavity are expected to be stronger than for the wider, secondary end.

The electrostatic energy term generally consists of large compensatory contributions: the solute-solute and solventsolvent electrostatic energies each contribute about 20-40 $\mathrm{kcal} / \mathrm{mol}$ toward favorable binding enthalpy, which is opposed by an unfavorable solute-solvent term in the range of 50-80 $\mathrm{kcal} / \mathrm{mol}$ (Table S16). An exception to this is ammonium's binding in the secondary orientation, in which the magnitude of these energies is in the range of $5-20 \mathrm{kcal} / \mathrm{mol}$. In general, the compensatory nature of the solute-solute and solvent-solvent energies with the solute-solvent energy can be understood as the host and guest sacrificing favorable interactions with the solvent in order to form favorable interactions with each other. The magnitude of these energies and the apparent aberration of the ammoniums in the secondary orientation seem to be correlated with the degree of desolvation that the polar headgroup of the guest encounters upon binding. Indeed, an overlay of 400 trajectory snapshots showing the positions of the ammonium nitrogen or carboxylate oxygen relative to the $\mathrm{CD}$ host reveals a noticeable difference in the placement of the guest polar group (Figure 6). In both orientations, the ammonium guests prefer placement of their polar group further into the solvent relative to the placement of carboxylate guests. This roughly correlates with the magnitude of the electrostatic terms (Table S16).

Inspection of the decomposed enthalpy and entropy components for guest binding (Tables S4, S6-S8) provides some insight into how the water model force field parameters contribute to binding. For simplicity, we focus on the $n$-alkyl ammonium and carboxylate guests (Figure 7 and Figures S4S6). The electrostatic component of the binding enthalpy differs the most between the water models. For example, compared to Q4RG-TIP3P (Figure 7), the electrostatic term for Q4RG-OPC increases in unfavorability by $2-6 \mathrm{kcal} / \mathrm{mol}$ (Figure S6), making binding of carboxylates in the secondary orientation unfavorable. The entropic term compensates for this by becoming uniformly favorable. It is likely that the magnitude of the water partial charges is the primary cause for this effect. For TIP3P, SPC/E, TIP4Pew, and OPC, the hydrogen charge values are $0.417,0.424,0.524$, and 0.679 , respectively. The favorability of both the mean electrostatic term and mean entropic contribution seem to correlate well with these values, with $R^{2}$ greater than 0.85 for the $n$-alkyl ammonium and carboxylate test set (Figure S10).

The link between the LJ contribution and the Lennard-Jones terms of the water model is less obvious. The TIP3P water model has the smallest $\sigma$ and $\varepsilon$ values $(3.151 \AA$ and $0.152 \mathrm{kcal} /$ mol, respectively) and tends to yield more favorable average LJ contributions than the other water models by approximately 0.7 $\mathrm{kcal} / \mathrm{mol}$ for both the primary and secondary orientations (Tables S4, S6-S8). The TIP4Pew, SPC/E, and OPC water models all have $\sigma$ values in the $3.16 \AA$ range; however, whereas TIP4Pew and SPC/E have an $\varepsilon$ value between 0.155 and 0.162 $\mathrm{kcal} / \mathrm{mol}$, OPC deviates significantly with a value of $0.213 \mathrm{kcal} /$ mol. This deviation does not appear to increase the LJ favorability for OPC, as its contribution is actually less favorable on average than TIP4Pew and SCP/E by about $0.1-0.3 \mathrm{kcal} /$ mol. The net effect of changing the water LJ model's parameters presumably reflects a balance between a greater loss of favorable solute-solvent interactions on binding, along with a greater gain of favorable solvent-solvent interactions as water is displaced from the surfaces of the solutes into the bulk.

Quality Assurance Testing. We performed several tests to evaluate whether our simulation setup and analysis is robust and precise. First, we evaluated whether our uncertainty estimates were reasonable. We created three smaller replicate simulation sets (Q4RG-TIP3P-sm1, Q4RG-TIP3P-sm2, Q4RG-TIP3P-sm3) with exactly identical simulation parameters as Q4RG-TIP3P, except that they used different random placements of solvent molecules during system building and different random number seeds during production simulations, 
and comprised just a subset of 15 host-guest pairs out of the full set of 43. Comparison of the replicate binding free energies and binding enthalpies showed excellent agreement (RMSD < $\left.0.39 \mathrm{kcal} / \mathrm{mol}, R^{2}>0.95\right)$ with the original simulation set (Figure S17).

We then tested whether our "wall restraints" - restraints which only impose a force on a bound guest molecule when it strays too far into the bulk solvent during the attachment phase of the APR procedure - significantly affected the binding calculations. The distance at which the wall restraint potential becomes nonzero was chosen to be far enough away from the binding site that we anticipated no impact on the calculations. However, to test this, we shortened the distance at which the restraints took effect by $1 \AA$ and tested the same 15 host-guest pairs that were studied in the replicates described above, thus forming the Q4RG-TIP3P-shw simulation set. The results again showed excellent agreement $\left(\mathrm{RMSD}<0.42 \mathrm{kcal} / \mathrm{mol}, R^{2}>\right.$ 0.93, Figure S17), suggesting that the restraints were not impacting the calculations.

Finally, although all reported binding free energy calculations in this work used the thermodynamic integration method, we also computed free energies using the MBAR method. ${ }^{41}$ The two methods were essentially indistinguishable, with the average absolute deviation of $0.14 \mathrm{kcal} / \mathrm{mol}$ and a maximum of $0.37 \mathrm{kcal} / \mathrm{mol}$ for the Q4RG-TIP3P simulation set.

\section{DISCUSSION}

The results described here represent, to the best of our knowledge, the most comprehensive host-guest validation panel examined to date with explicit solvent free energy methods. We evaluated several water models, host and guest force fields, ion parameters, and charge assignment methods in order to improve our understanding of the accuracy of currently available force field parameters. This effort relied on the significant performance advantage afforded by GPU processors, along with a highly automated implementation of our APR method, in order to deploy the thousands of simulations which produced this work. We expect investigations of similar scope to become more commonplace as efforts to improve force fields using automated optimization schemes become tractable for larger systems. ${ }^{36,63}$ The following discussion highlights results from this work which could inform such future endeavors.

Force Field Selection and Development. It appears that none of the force field combinations we tested is clearly superior to the others, and the thermodynamic values they yield are remarkably wide-ranging. For example, the binding enthalpy of octanoate to $\alpha \mathrm{CD}$ ("a-oct") has a range greater than $8 \mathrm{kcal} /$ mol depending on the force field combination choice. Surprisingly, the arguably crude AM1-BCC/GAFF host force field with AM1-BCC/GAFF guest parameters (i.e., the BGBGTIP3P simulation set) outperforms all other force field combinations we tested at calculating binding free energies; but it performs poorly for binding enthalpies. However, conformational analysis of the BGBG-TIP3P simulation set casts doubt on the accuracy with which it captures the conformational preferences of the CDs and thus suggests that some of the thermodynamic results it yields may be right for the wrong reasons. In contrast, the Q4RG-TIP4Pew combination does remarkably well at calculating binding enthalpies but only does moderately well for free energies.

The fact that the correlation with experiment tends to be higher if one restricts attention to a subset of guests with the same functional group, as opposed to considering all guests together (Figure 3), suggests that force field errors could combine in unpredictable ways for more complicated molecules with multiple functional groups. Depending on the exact binding site composition of the host and guest, or protein and ligand, such errors could combine to seriously overestimate or underestimate the experimental binding thermodynamics and lead to difficulties in correctly ranking ligands by affinity. Alternatively, the errors could combine in such a way as to cancel out and yield good agreement with experiment. Unsurprisingly, this points to the critical importance of diverse training and testing sets. It will likely become increasingly important to collaborate with experimentalists in generating these data sets, rather than relying on existing data which were not developed specifically to test and guide force field development.

Entropy-enthalpy compensation appears to play a role in mitigating how changes in parameters propagate to changes in the binding free energy. We observed two such mechanisms. First, although drastic changes in enthalpy were observed when moving from the TIP3P to the OPC water model, these were accompanied by much more modest changes in free energy. In this case, it seems that entropy-enthalpy compensation likely operated through changes in the water structure driven by large changes in the magnitude of the water charges. Second, we found that, when moving from the Q4MD-CD host force field to AM1-BCC/GAFF (i.e., Q4RG-TIP3P to BGBG-TIP3P), the primary difference was found in the dihedral parameters of the CD hosts, and thus entropy-enthalpy likely operated through combined structural changes in the host and subsequently changes to the structured water within the host cavity.

Binding free energy studies which investigate charged ligands are comparatively rare, possibly due to inherent difficulty or perhaps technical challenges associated with commonly used alchemical approaches. ${ }^{64}$ The marked difference in mean signed error for the binding free energy of each guest class, which have different net charges, suggests that there might be problems with the partial charge assignments. One possibility is that the RESP charge approach, in which partial charges are chosen to reproduce gas phase QM electrostatic potentials, does not fully take into account the electronic polarization of water which provides electronic screening of solute charges. Leontyev and Stuchebrukhov have suggested that ionic solutes, in particular, should have their gas phase partial charges scaled by 0.7 in order to account for water polarization. ${ }^{65,66}$ Such proposals will be of interest to future studies.

Comparison with Other Host-Guest Calculations. The results of this study are broadly consistent with other hostguest calculations we have performed previously. ${ }^{36,40,58,60,67}$ The binding free energy is generally overestimated, albeit much more modestly for CD than for CB7 hosts. The OPC water model significantly shifts the binding enthalpy in the positive direction, which we have observed for other host-guest systems. $^{60,67}$ Additionally, as we observed with $\mathrm{CB} 7,{ }^{60}$ valence (bonded) terms can contribute significantly to binding.

On the other hand, significant differences also emerge. For example, the OPC water model looks very promising for the CB7 system, where binding free energies and enthalpies with the TIP3P water model had been significantly overestimated, so that OPC's positive shift in these values brought them closer to experiment (Gao et al. ${ }^{60}$ and unpublished data). In contrast, the present results, which focus on CDs, show that, while the OPC model does yield a positive shift in the binding free energy 
relative to TIP3P, bringing those numbers closer to experiment, the large positive shift in binding enthalpy greatly increases its deviation from experiment. Furthermore, for octa-acid hosts, ${ }^{67}$ the positive shift in enthalpy when moving from TIP3P to OPC is accompanied by a small negative shift in the binding free energy, moving those values further from experiment. Evidently, the influence of the water model on the agreement of the binding free energy and the enthalpy with experiment are system-dependent.

Guest Orientation. We were surprised and perplexed that the Q4MD calculations predicted that ammonium guests prefer to bind CDs in the primary orientation. Although there is not much evidence for the conventional viewpoint that $n$-alkyl polar groups will orient out of the secondary cavity of CDs, this does seem to be generally accepted. ${ }^{37,62,68}$ The NMR data published by Rekharsky et al. ${ }^{37}$ does not show any indication that ammoniums might be binding in the primary orientation, although it does not strongly point to binding in the secondary orientation either.

We considered whether the shift in the linear regressions between calculated and experimental binding free energy values for ammoniums and carboxylates (see Figure 3) might reflect an incorrect binding orientation of the ammoniums. However, if one ignores the primary orientation data and only uses the results from the secondary orientation, the deviation between the guest classes grows even larger, because the secondary orientation is of lower affinity than the primary orientation, and ammoniums already tended to be assigned lower affinities than carboxylates.

Directions. The present study demonstrates that hostguest systems can be used to systematically characterize and compare the accuracy of force fields in the calculation of binding thermodynamics. The present data set, and future expansions of it, highlights and should ultimately help resolve a number of questions, such as how can one selectively shift the binding affinity of host-guest pairs, depending on their polar functional groups, and what strategies can be employed in force field optimization to account for the phenomenon of entropyenthalpy compensation, which complicates the selection of parameters that will accurately replicate both binding free energies and enthalpies. With increasing computer power, it should be possible to incorporate such data into an overall force field optimization strategy, which uses heterogeneous data sets that also include, for example, quantum mechanical calculations, pure liquid properties, and crystallographic data. Our lab will continue to investigate these questions as part of the larger community effort to improve force fields and thus advance the accuracy of binding calculations.

\section{ASSOCIATED CONTENT}

\section{S Supporting Information}

The Supporting Information is available free of charge on the ACS Publications website at DOI: 10.1021/acs.jctc.7b00359.

Figures S1-S18 (PDF)

Tables S1-S16 (XLSX)

\section{AUTHOR INFORMATION}

\section{Corresponding Author}

*E-mail: mgilson@ucsd.edu.

ORCID

Niel M. Henriksen: 0000-0002-7916-0757

Michael K. Gilson: 0000-0002-3375-1738

\section{Funding}

This work used computational resources from both the Triton Shared Computing Cluster at UCSD and the Extreme Science and Engineering Discovery Environment (XSEDE), which is supported by the National Science Foundation grant no. ACI1053575. M.K.G. acknowledges funding from the National Institute of General Medical Sciences (GM61300).

\section{Notes}

The contents of this paper are solely the responsibility of the authors and do not necessarily represent the official views of the NIH.

The authors declare the following competing financial interest(s): M.K.G. has an equity interest in and is a cofounder and scientific advisor of VeraChem LLC.

\section{REFERENCES}

(1) Robertson, M. J.; Tirado-Rives, J.; Jorgensen, W. L. Improved Peptide and Protein Torsional Energetics with the OPLS-AA Force Field. J. Chem. Theory Comput. 2015, 11 (7), 3499-3509.

(2) Harder, E.; Damm, W.; Maple, J.; Wu, C.; Reboul, M.; Xiang, J. Y.; Wang, L.; Lupyan, D.; Dahlgren, M. K.; Knight, J. L.; Kaus, J. W.; Cerutti, D. S.; Krilov, G.; Jorgensen, W. L.; Abel, R.; Friesner, R. A. OPLS3: A Force Field Providing Broad Coverage of Drug-like Small Molecules and Proteins. J. Chem. Theory Comput. 2016, 12 (1), 281296.

(3) Case, D. A.; Betz, R. M.; Botello-Smith, W.; Cerutti, D. S.; Cheatham, T. E., III; Darden, T. A.; Duke, R. E.; Giese, T. J.; Gohlke, H.; Goetz, A. W.; Homeyer, N.; Izadi, S.; Janowski, P.; Kaus, J.; Kovalenko, A.; Lee, T. S.; LeGrand, S.; Li, P.; Lin, C.; Luchko, T.; Luo, R.; Madej, B.; Mermelstein, D.; Merz, K. M.; Monard, G.; Nguyen, H.; Nguyen, H. T.; Omelyan, I.; Onufriev, A.; Roe, D. R.; Roitberg, A.; Sagui, C.; Simmerling, C. L.; Swails, J.; Walker, R. C.; Wang, J.; Wolf, R. M.; Wu, X.; Xiao, L.; York, D. M.; Kollman, P. A. AMBER 16; University of California: San Francisco, 2016.

(4) Vanommeslaeghe, K.; Hatcher, E.; Acharya, C.; Kundu, S.; Zhong, S.; Shim, J.; Darian, E.; Guvench, O.; Lopes, P.; Vorobyov, I.; Mackerell, A. D. CHARMM General Force Field: A Force Field for Drug-like Molecules Compatible with the CHARMM All-Atom Additive Biological Force Fields. J. Comput. Chem. 2010, 31 (4), 671-690.

(5) Best, R. B.; Zhu, X.; Shim, J.; Lopes, P. E. M.; Mittal, J.; Feig, M.; MacKerell, A. D. Optimization of the Additive CHARMM All-Atom Protein Force Field Targeting Improved Sampling of the Backbone $\Phi$, $\Psi$ and Side-Chain $\chi 1$ and $\chi 2$ Dihedral Angles. J. Chem. Theory Comput. 2012, 8 (9), 3257-3273.

(6) Reif, M. M.; Hünenberger, P. H.; Oostenbrink, C. New Interaction Parameters for Charged Amino Acid Side Chains in the GROMOS Force Field. J. Chem. Theory Comput. 2012, 8 (10), 37053723.

(7) Ploetz, E. A.; Smith, P. E. A Kirkwood-Buff Force Field for the Aromatic Amino Acids. Phys. Chem. Chem. Phys. 2011, 13 (40), 18154-18167.

(8) Baker, C. M.; Darian, E.; MacKerell, A. D., Jr. Chapter 3: Towards Biomolecular Simulations with Explicit Inclusion of Polarizability: Development of a CHARMM Polarizable Force Field Based on the Classical Drude Oscillator Model. In Innovations in Biomolecular Modeling and Simulations: Volume 1; 2012; pp 23-50.

(9) Lemkul, J. A.; MacKerell, A. D. Balancing the Interactions of $\mathrm{Mg} 2+$ in Aqueous Solution and with Nucleic Acid Moieties For a Polarizable Force Field Based on the Classical Drude Oscillator Model. J. Phys. Chem. B 2016, 120 (44), 11436-11448.

(10) Friesner, R. A. Modeling Polarization in Proteins and Proteinligand Complexes: Methods and Preliminary Results; Peptide Solvation and HBonds; Academic Press: 2005; Vol. 72, pp 79-104.

(11) Wang, Z.-X.; Zhang, W.; Wu, C.; Lei, H.; Cieplak, P.; Duan, Y. Strike a Balance: Optimization of Backbone Torsion Parameters of 
AMBER Polarizable Force Field for Simulations of Proteins and Peptides. J. Comput. Chem. 2006, 27 (6), 781-790.

(12) Shi, Y.; Xia, Z.; Zhang, J.; Best, R.; Wu, C.; Ponder, J. W.; Ren, P. Polarizable Atomic Multipole-Based AMOEBA Force Field for Proteins. J. Chem. Theory Comput. 2013, 9 (9), 4046-4063.

(13) Duke, R. E.; Starovoytov, O. N.; Piquemal, J.-P.; Cisneros, G. A. GEM*: A Molecular Electronic Density-Based Force Field for Molecular Dynamics Simulations. J. Chem. Theory Comput. 2014, 10 (4), 1361-1365.

(14) Cole, D. J.; Vilseck, J. Z.; Tirado-Rives, J.; Payne, M. C.; Jorgensen, W. L. Biomolecular Force Field Parameterization via Atoms-in-Molecule Electron Density Partitioning. J. Chem. Theory Comput. 2016, 12 (5), 2312-2323.

(15) Grimme, S. A General Quantum Mechanically Derived Force Field (QMDFF) for Molecules and Condensed Phase Simulations. J. Chem. Theory Comput. 2014, 10 (10), 4497-4514.

(16) Monti, S.; Corozzi, A.; Fristrup, P.; Joshi, K. L.; Shin, Y. K.; Oelschlaeger, P.; van Duin, A. C. T.; Barone, V. Exploring the Conformational and Reactive Dynamics of Biomolecules in Solution Using an Extended Version of the Glycine Reactive Force Field. Phys. Chem. Chem. Phys. 2013, 15 (36), 15062-15077.

(17) Gao, J.; Truhlar, D. G.; Wang, Y.; Mazack, M. J. M.; Löffler, P.; Provorse, M. R.; Rehak, P. Explicit Polarization: A Quantum Mechanical Framework for Developing Next Generation Force Fields. Acc. Chem. Res. 2014, 47 (9), 2837-2845.

(18) Jorgensen, W. L.; Chandrasekhar, J.; Madura, J. D.; Impey, R. W.; Klein, M. L. Comparison of Simple Potential Functions for Simulating Liquid Water. J. Chem. Phys. 1983, 79 (2), 926-935.

(19) Horn, H. W.; Swope, W. C.; Pitera, J. W.; Madura, J. D.; Dick, T. J.; Hura, G. L.; Head-Gordon, T. Development of an Improved Four-Site Water Model for Biomolecular Simulations: TIP4P-Ew. J. Chem. Phys. 2004, 120 (20), 9665-9678.

(20) Berendsen, H. J. C.; Grigera, J. R.; Straatsma, T. P. The Missing Term in Effective Pair Potentials. J. Phys. Chem. 1987, 91 (24), 62696271.

(21) Izadi, S.; Anandakrishnan, R.; Onufriev, A. V. Building Water Models: A Different Approach. J. Phys. Chem. Lett. 2014, 5 (21), 3863-3871.

(22) Wang, L.-P.; Martinez, T. J.; Pande, V. S. Building Force Fields: An Automatic, Systematic, and Reproducible Approach. J. Phys. Chem. Lett. 2014, 5 (11), 1885-1891.

(23) Piana, S.; Donchev, A. G.; Robustelli, P.; Shaw, D. E. Water Dispersion Interactions Strongly Influence Simulated Structural Properties of Disordered Protein States. J. Phys. Chem. B 2015, 119 (16), 5113-5123.

(24) Wang, L.-P.; Head-Gordon, T.; Ponder, J. W.; Ren, P.; Chodera, J. D.; Eastman, P. K.; Martinez, T. J.; Pande, V. S. Systematic Improvement of a Classical Molecular Model of Water. J. Phys. Chem. B 2013, 117 (34), 9956-9972.

(25) Hess, B.; van der Vegt, N. F. A. Hydration Thermodynamic Properties of Amino Acid Analogues: A Systematic Comparison of Biomolecular Force Fields and Water Models. J. Phys. Chem. B 2006, 110 (35), 17616-17626.

(26) Mobley, D. L.; Bayly, C. I.; Cooper, M. D.; Shirts, M. R.; Dill, K. A. Small Molecule Hydration Free Energies in Explicit Solvent: An Extensive Test of Fixed-Charge Atomistic Simulations. J. Chem. Theory Comput. 2009, 5 (2), 350-358.

(27) Galindo-Murillo, R.; Robertson, J. C.; Zgarbová, M.; Šponer, J.; Otyepka, M.; Jurečka, P.; Cheatham, T. E. Assessing the Current State of Amber Force Field Modifications for DNA. J. Chem. Theory Comput. 2016, 12 (8), 4114-4127.

(28) Bergonzo, C.; Henriksen, N. M.; Roe, D. R.; Cheatham, T. E. Highly Sampled Tetranucleotide and Tetraloop Motifs Enable Evaluation of Common RNA Force Fields. RNA 2015, 21 (9), $1578-1590$.

(29) Beauchamp, K. A.; Lin, Y.-S.; Das, R.; Pande, V. S. Are Protein Force Fields Getting Better? A Systematic Benchmark on 524 Diverse NMR Measurements. J. Chem. Theory Comput. 2012, 8 (4), 14091414.
(30) Maier, J. A.; Martinez, C.; Kasavajhala, K.; Wickstrom, L.; Hauser, K. E.; Simmerling, C. ff14SB: Improving the Accuracy of Protein Side Chain and Backbone Parameters from ff99SB. J. Chem. Theory Comput. 2015, 11 (8), 3696-3713.

(31) Wickstrom, L.; He, P.; Gallicchio, E.; Levy, R. M. Large Scale Affinity Calculations of Cyclodextrin Host-Guest Complexes: Understanding the Role of Reorganization in the Molecular Recognition Process. J. Chem. Theory Comput. 2013, 9 (7), 3136-3150.

(32) Zhang, H.; Yin, C.; Yan, H.; van der Spoel, D. Evaluation of Generalized Born Models for Large Scale Affinity Prediction of Cyclodextrin Host-Guest Complexes. J. Chem. Inf. Model. 2016, 56 (10), 2080-2092.

(33) Muddana, H. S.; Varnado, C. D.; Bielawski, C. W.; Urbach, A. R.; Isaacs, L.; Geballe, M. T.; Gilson, M. K. Blind Prediction of Hostguest Binding Affinities: A New SAMPL3 Challenge. J. Comput.-Aided Mol. Des. 2012, 26 (5), 475-487.

(34) Muddana, H. S.; Fenley, A. T.; Mobley, D. L.; Gilson, M. K. The SAMPL4 Host-guest Blind Prediction Challenge: An Overview. J. Comput.-Aided Mol. Des. 2014, 28 (4), 305-317.

(35) Yin, J.; Henriksen, N. M.; Slochower, D. R.; Shirts, M. R.; Chiu, M. W.; Mobley, D. L.; Gilson, M. K. Overview of the SAMPL5 Hostguest Challenge: Are We Doing Better? J. Comput.-Aided Mol. Des. 2017, 31, 1-19.

(36) Yin, J.; Fenley, A. T.; Henriksen, N. M.; Gilson, M. K. Toward Improved Force-Field Accuracy through Sensitivity Analysis of HostGuest Binding Thermodynamics. J. Phys. Chem. B 2015, 119 (32), 10145-10155.

(37) Rekharsky, M. V.; Mayhew, M. P.; Goldberg, R. N.; Ross, P. D.; Yamashoji, Y.; Inoue, Y. Thermodynamic and Nuclear Magnetic Resonance Study of the Reactions of $\alpha$ - and $\beta$-Cyclodextrin with Acids, Aliphatic Amines, and Cyclic Alcohols. J. Phys. Chem. B 1997, 101 (1), 87-100.

(38) Mobley, D. L.; Gilson, M. K. Predicting Binding Free Energies: Frontiers and Benchmarks. Annu. Rev. Biophys. 2017, 46 (1), 531-558.

(39) Rekharsky, M.; Inoue, Y. Chiral Recognition Thermodynamics of $\beta$-Cyclodextrin: The Thermodynamic Origin of Enantioselectivity and the Enthalpy-Entropy Compensation Effect. J. Am. Chem. Soc. 2000, 122 (18), 4418-4435.

(40) Henriksen, N. M.; Fenley, A. T.; Gilson, M. K. Computational Calorimetry: High-Precision Calculation of Host-Guest Binding Thermodynamics. J. Chem. Theory Comput. 2015, 11 (9), 4377-4394.

(41) Shirts, M. R.; Chodera, J. D. Statistically Optimal Analysis of Samples from Multiple Equilibrium States. J. Chem. Phys. 2008, 129 (12), 124105.

(42) Flyvbjerg, H.; Petersen, H. G. Error Estimates on Averages of Correlated Data. J. Chem. Phys. 1989, 91 (1), 461-466.

(43) Zhang, H.; Tan, T.; Hetényi, C.; van der Spoel, D. Quantification of Solvent Contribution to the Stability of Noncovalent Complexes. J. Chem. Theory Comput. 2013, 9 (10), 4542-4551.

(44) Joung, I. S.; Cheatham, T. E. Determination of Alkali and Halide Monovalent Ion Parameters for Use in Explicitly Solvated Biomolecular Simulations. J. Phys. Chem. B 2008, 112 (30), 90209041.

(45) Steinbrecher, T.; Latzer, J.; Case, D. A. Revised AMBER Parameters for Bioorganic Phosphates. J. Chem. Theory Comput. 2012, 8 (11), 4405-4412.

(46) Cézard, C.; Trivelli, X.; Aubry, F.; Djedaïni-Pilard, F.; Dupradeau, F.-Y. Molecular Dynamics Studies of Native and Substituted Cyclodextrins in Different Media: 1. Charge Derivation and Force Field Performances. Phys. Chem. Chem. Phys. 2011, 13 (33), $15103-15121$.

(47) Kirschner, K. N.; Woods, R. J. Quantum Mechanical Study of the Nonbonded Forces in Water-Methanol Complexes. J. Phys. Chem. A 2001, 105 (16), 4150-4155.

(48) Kirschner, K. N.; Woods, R. J. Solvent Interactions Determine Carbohydrate Conformation. Proc. Natl. Acad. Sci. U. S. A. 2001, 98 (19), 10541-10545. 
(49) Basma, M.; Sundara, S.; Çalgan, D.; Vernali, T.; Woods, R. J. Solvated Ensemble Averaging in the Calculation of Partial Atomic Charges. J. Comput. Chem. 2001, 22 (11), 1125-1137.

(50) Jakalian, A.; Bush, B. L.; Jack, D. B.; Bayly, C. I. Fast, Efficient Generation of High-Quality Atomic Charges. AM1-BCC Model: I. Method. J. Comput. Chem. 2000, 21 (2), 132-146.

(51) Jakalian, A.; Jack, D. B.; Bayly, C. I. Fast, Efficient Generation of High-Quality Atomic Charges. AM1-BCC Model: II. Parameterization and Validation. J. Comput. Chem. 2002, 23 (16), 1623-1641.

(52) Wang, J.; Wolf, R. M.; Caldwell, J. W.; Kollman, P. A.; Case, D. A. Development and Testing of a General Amber Force Field. J. Comput. Chem. 2004, 25 (9), 1157-1174.

(53) Vanquelef, E.; Simon, S.; Marquant, G.; Garcia, E.; Klimerak, G.; Delepine, J. C.; Cieplak, P.; Dupradeau, F.-Y. R.E.D. Server: A Web Service for Deriving RESP and ESP Charges and Building Force Field Libraries for New Molecules and Molecular Fragments. Nucleic Acids Res. 2011, 39 (suppl_2), W511-W517.

(54) Harvey, M. J.; Giupponi, G.; Fabritiis, G. D. ACEMD: Accelerating Biomolecular Dynamics in the Microsecond Time Scale. J. Chem. Theory Comput. 2009, 5 (6), 1632-1639.

(55) Hopkins, C. W.; Le Grand, S.; Walker, R. C.; Roitberg, A. E. Long Time Step Molecular Dynamics through Hydrogen Mass Repartitioning. J. Chem. Theory Comput. 2015, 11 (4), 1864-1874.

(56) Loncharich, R. J.; Brooks, B. R.; Pastor, R. W. Langevin Dynamics of Peptides: The Frictional Dependence of Isomerization Rates of N-Acetylalanyl-N'-Methylamide. Biopolymers 1992, 32 (5), 523-535.

(57) Åqvist, J.; Wennerström, P.; Nervall, M.; Bjelic, S.; Brandsdal, B. O. Molecular Dynamics Simulations of Water and Biomolecules with a Monte Carlo Constant Pressure Algorithm. Chem. Phys. Lett. 2004, 384 (4-6), 288-294.

(58) Fenley, A. T.; Henriksen, N. M.; Muddana, H. S.; Gilson, M. K. Bridging Calorimetry and Simulation through Precise Calculations of Cucurbituril-Guest Binding Enthalpies. J. Chem. Theory Comput. 2014, 10 (9), 4069-4078.

(59) Bergonzo, C.; Cheatham, T. E. Improved Force Field Parameters Lead to a Better Description of RNA Structure. J. Chem. Theory Comput. 2015, 11 (9), 3969-3972.

(60) Gao, K.; Yin, J.; Henriksen, N. M.; Fenley, A. T.; Gilson, M. K. Binding Enthalpy Calculations for a Neutral Host-Guest Pair Yield Widely Divergent Salt Effects across Water Models. J. Chem. Theory Comput. 2015, 11 (10), 4555-4564.

(61) Bayly, C. I.; Cieplak, P.; Cornell, W.; Kollman, P. A. A WellBehaved Electrostatic Potential Based Method Using Charge Restraints for Deriving Atomic Charges: The RESP Model. J. Phys. Chem. 1993, 97 (40), 10269-10280.

(62) Connors, K. A. The Stability of Cyclodextrin Complexes in Solution. Chem. Rev. 1997, 97 (5), 1325-1358.

(63) Wang, L.-P.; McKiernan, K. A.; Gomes, J.; Beauchamp, K. A.; Head-Gordon, T.; Rice, J. E.; Swope, W. C.; Martínez, T. J.; Pande, V. S. Building a More Predictive Protein Force Field: A Systematic and Reproducible Route to AMBER-FB15. J. Phys. Chem. B 2017, 121 (16), 4023-4039.

(64) Rocklin, G. J.; Mobley, D. L.; Dill, K. A.; Hünenberger, P. H. Calculating the Binding Free Energies of Charged Species Based on Explicit-Solvent Simulations Employing Lattice-Sum Methods: An Accurate Correction Scheme for Electrostatic Finite-Size Effects. J. Chem. Phys. 2013, 139 (18), 184103.

(65) Leontyev, I.; Stuchebrukhov, A. Accounting for Electronic Polarization in Non-Polarizable Force Fields. Phys. Chem. Chem. Phys. 2011, 13 (7), 2613-2626.

(66) Leontyev, I. V.; Stuchebrukhov, A. A. Polarizable Molecular Interactions in Condensed Phase and Their Equivalent Nonpolarizable Models. J. Chem. Phys. 2014, 141 (1), 014103.

(67) Yin, J.; Henriksen, N. M.; Slochower, D. R.; Gilson, M. K. The SAMPL5 Host-guest Challenge: Computing Binding Free Energies and Enthalpies from Explicit Solvent Simulations by the Attach-PullRelease (APR) Method. J. Comput.-Aided Mol. Des. 2017, 31 (1), 133145 .
(68) Rekharsky, M. V.; Inoue, Y. Complexation Thermodynamics of Cyclodextrins. Chem. Rev. 1998, 98 (5), 1875-1918. 